\title{
Uber den Mechanismus des Nahrungserwerbs der Tunicaten, speziell der Ascidien ${ }^{1}$ )
}

\author{
Von Erika und Bernhard Werner \\ Aus der Biologischen Anstalt Helgoland, List auf Sylt, \\ in der Bundesforschungsanstalt für Fischerei \\ (Mit 15 Abbildungen und 1 Tabelle im Text)
}

Inhaltsübersicht

A. Einleitung S. 57 - B. Frühere Untersuchungen S. 58 - C. Der Mechanismus des Nahrungserwerbs S. 59 - I. Die Bildung des Schleimfilters S. 61 - II. Der Transportvorgang. die eigentliche Filtrationsphase S. 66 - III. Der Sammelvorgang S. 75 - IV. Die Aufnahme der Futterwurst in den Oesophagus S. $80-$ D. Besprechung der Ergebnisse S. 81 - E. Zusammenfassung S. 89 - F. Literaturverzeichnis S. 90.

\section{A. Einleitung}

Die Tunicaten gehören hinsichtlich ihrer Ernährungsbiologie zu der großen Gruppe mariner Tiere, die ihre Nahrung, Plankton und Detritus, durch Filtration des Atemwasserstromes gewinnen. Das trifft in gleicher Weise für die festsitzenden Formen (Ascidiacea) zu, wie auch für die freischwimmenden (Copelata und Thaliacea, sowie die Pyrosomida unter den Ascidiacea). Die Grundvorgänge des Nahrungserwerbs sind stets: a) die aktive Erzeugung des Wasserstromes und b) seine Filtration. Dementsprechend stimmt auch der eigentliche Mechanismus des Nahrungserwerbs, der als Wasserstromfiltration gekennzeichnet werden kann, bei diesen nach Morphologie und Lebensweise unterschiedlichen Gruppen überein.

Die im folgenden mitgeteilten Beobachtungen über den Nahrungserwerb der Ascidien gehen aus von Untersuchungen über den Nahrungserwerb einer besonders spezialisierten Gruppe der Wasserstromfiltrierer unter den Prosobranchiern, der Familie Calyptraeidae (Werner 1951, 1953 a, b). Die Ergebnisse legten nahe, den Nahrungserwerb auch bei Wasserstromfiltrierern aus anderen Tierstämmen einer vergleichenden Analyse zu unterziehen, soweit sie mit den Hilfsmitteln der Lebendbeobachtung und des Suspensionsversuches durchführbar war. Das erschien von vornherein als aussichtsreich für die Ascidien, die einmal diesem Ernährungstyp ausschließlich angehören und andererseits mit einzelnen Arten wegen ihrer Größe und ihrer im erwachsenen oder doch im jugendlichen Zustand großen Durchsichtigkeit für eine Lebendbeobachtung besonders geeignet sein mußten.

Die Untersuchungen wurden im Frühjahr 1951 von E. WerNer in Neapel an Ciona intestinalis L. begonnen; dabei konnten die Einzelphasen des Filtra-

1) Herrn Prof. W. v, Buddenbrock, Mainz, zum 70. Geburtstag gewidmet. 
tionsvorganges und die Bedeutung der beteiligten Organe im wesentlichen klargestellt werden. Die Ergebnisse wurden in der Folgezeit von B. WERnER für Clavelina lepadiformis O. F. MüLLER bestätigt. Es gelang, mehrere Kolonien dieser Art im Aquarium anzusiedeln, die sich in jedem Jahr aus Winterknospen neu entwickeln, so daß genügend Material aller Altersstufen zur Verfügung stand. Wegen ihres einfachen und übersichtlichen Baues kann diese besonders schöne und durchsichtige Form geradezu als lebendes Schema einer Ascidie bezeichnet werden und bietet sidh für die Darstellung des Nahrungserwerbs besonders an.

Der Deutschen Forschungsgemeinschaft haben wir für eine Reisebeihilfe zu danken, die uns die Durchführung auch dieser Untersudungen an der Zoologischen Station Neapel ermöglichte. Ebenso möchten wir auch an dieser Stelle dem Direktor der Station, Herrn Prof. R. DoHRN, für dic Bereitstellung eines Arbeitsplatzes, sowie den anderen Herren der Station für die vielseitige Unterstützung und die Mithilfe beì der Materialbeschaffung danken. Schließlich möchten wir auch Herrn Prof. C. Schlieper, Kiel, für die Beschaffung lebender Ciona unseren Dank aussprechen.

\section{B. Frühere Untersuchungen}

Die Tunicaten haben als phylogenetisch und systematisch wie auch biologisch wichtige Gruppe von jeher das besondere Interesse der Zoologen gefunden, doch liegen über den Nahrungserwerb nur wenige eingehende Untersuchungen vor. Die früheren Arbeiten über dieses Problem stehen in engem Zusammenhang mit der Frage nach der Bedeutung der Hypobranchialrinne oder des Endostyls, der ventralen Drüsenrinne des Kiemendarmes. Fol $(1872,1876)$ ist wohl der erste gewesen, der die Funktion des Endostyls und die Art des Nahrungserwerbs klar erkannt hat. Fols Ergebnisse, daß der Nahrungserwerb an den Schleim gebunden ist, der durch seine klebrige Beschaffenheit die Nahrungspartikel auffängt und vom Endostyl gebildet wird, und daß bei den Copelata und Thaliacea der Schleim im Kiemendarm die Form eines Trichters annimmt, sind heute noch unverändert gültig.

Rovle (1884) hat in seiner Monographie über Ciona intéstinalis auch die Organe des Kiemendarmes und ihre Funktion sehr eingehend untersucht und beschrieben; er stimmt mit den früheren Autoren hinsichtlich des allgemeinen Mechanismus des Nahrungserwerbs überein. Nur in der Funktion des Endostyls ist er gegenteiliger Auffassung. Roule hält den Endostyl nicht für ein schleimerzeugendes Drüsenorgan, sondern sieht seine Aufgabe lediglich in dem Transport und der Verteilung des Schleims, der nach seiner Meinung in erster Linie von der Neuraldrüse geliefert werden soll, eine Auffassung, die gegenüber den eindeutigen Ergebnissen von Fou als Rüdsshritt zu bezeichnen ist (vgl. Fenene 1933).

Orton (1918) hat den Nahrungserwerb der Ascidien im Vergleich zu dem von Wasserstromfiltrierern aus anderen Tierstämmen untersucht und vor allem den Transport der vom Schleim festgehaltenen Nahrungspartikel über die Flächen des Kiemendarmes geprüft (vgl. Heсhт 1918).

Eine eingehendere Darstellung des Mechanismus des Nahrungserwerbs der Dolioliden und Salpen hat FEDRLE (1921, 1923, 1933) gegeben, die in ihren Ergebnissen für die Salpen von CARLISLE (1950) bestätigt und vervollständigt wurde.

Der wichtigste neuere Beitrag über den Nahrungserwerb der Tunicaten liegt in der Arbeit von MacGinme (1939 b) vor, der speziell für die Ascidien gezeigt hat, daß nicht die Kieme, sondern die beiden vom Endostyl gebildeten und anschließend ständig über die rechte und linke Innenfläche des Kiemendarmes transportierten Schleimgewebe das eigentliche Filter darstellen. Das Fehlen von Abbildungen erschwert das Verständnis der Ergebnisse von MACGinirie, die überdies nach unseren eigenen Beobadhtungen hinsichtlich der Bedeutung der beteiligten Organe und der Einzelphasen des Nahrungserwerbs der Ergänzung bedürfen.

Schließlich müssen noch die neucren Arbeiten von Hoyle $(1952,1953)$ genannt werden, der auf die Bedeutung der spontanen rhythmischen Kontraktionen für die Wassererneuerung und damit für den Nahrungserwerb der Ascidien aufmerksam gemacht hat. (Für die allgemeinere Behandlung des Nahrungserwerbs der Tunicaten nach den älteren Literaturangaben vgl. u. a. Ihle 1935, Huus 193\%, Berrili. 1950, Hagmeter 1951). 


\section{Der Mechanismus des Nahrungserwerbs}

In ähnlicher Weise, wie von Werner (1953 a) für den Mechanismus des Nahrungserwerbs der Calyptraeidae beschrieben wurde, lassen sich auch bei den Ascidien mehrere Einzelphasen und Teilvorgänge unterscheiden, die in ihrem koordinierten Zusammenwirken die Gesamtleistung des Nahrungserwerbs ergeben. Wie eingangs kurz erwähnt wurde, ist der Grundvorgang die aktive Erzeugung des Wasserstromes, der als Ingestionsstrom durch den Ingestions- oder Branchialsipho in den Kiemendarm eintritt, die Kiemenspalten durchströmt und aus dem Peribranchialraum, der sich auf der Dorsalseite zum Atrial- oder Kloakalraum erweitert, durch den Egestionsoder Atrialsipho wieder nach außen austritt. Der Wasserstrom dient primär dem Gasaustausch und wird erzeugt durch den Cilienschlag der Wimperzellen der Kiemenspalten, die eine rein hydromotorische Funktion haben. Gegenüber den Cilien der übrigen mit Wimperzellen besetzten Flächen und Organe des Kiemendarmes sind die Cilien der Wimperzellen der Kiemenspalten durch ihre besondere Länge ausgezeichnet, in der sie nur noch von den langen medianen Geißeln des Endostylgrundes übertroffen werden (s. u. S. 63 f. und Tab. 1). Die wirksame Phase des Vorwärtsschlages der Cilien an den Kiemenspalten ist von innen nach außen gerichtet; dabei wird in der Koordinierung des Wimperschlages aller Cilien eines Spaltes die bekannte Erscheinung der Metachronie sichtbar (vgl. Fedele 1923). Der Wimperschlag erzeugt so den Eindruck einer um den Spalt herumlaufenden Welle, deren Richtung bei allen Kiemenspalten die gleiche ist und von außen gesehen der Richtung des Uhrzeigers entgegengesetzt ist (MACGinitie $1939 \mathrm{~b}$ ). Daß in der rhythmischen Koordinierung des Schlages aller Cilien einer Kiemenspalte und des Wimperschlages bei allen Kiemenspalten ein höheres mechanisches Prinzip wirksam zu sein scheint, das mit dem zahlreicher Zahnradpumpen oder Turbinen vergleichbar sein dürfte, wurde von WerNer (1953 a) schon für die Calyptraeiden erwähnt, wo die gleiche Erscheinung zu beobachten ist. Durch die verschiedene Stärke und Richtung des In- und Egestionsstromes wird nach Hecht (1918) vermieden, daß die beiden Ströme sich vermischen, was in gleicher Weise für die Atmung und Ernährung bedeutungsvoll ist.

Nach den Untersuchungen von HoyLe $(1952,1953)$ stellen die spontanen rhythmischen Kontraktionen des Ascidienkörpers einen weiteren regulären Mechanismus zur Wassererneuerung dar. Bei mehreren Arten wurde nachgewiesen, daß sich der Körper unabhängig von äußeren Reizen in regelmäßigen Intervallen kontrahiert, so daß der größte Teil des Inhaltes des Branchial-, wie auch des Peribranchial- und Atrialraumes ruckartig ausgestoßen wird, wobei sich die Siphonen schließen. Wenn der Körper sich anschließend wieder ausdehnt und die Siphonen sich öffnen, wird automatisch frisches Wasser eingesogen. Die gesamte, in den Kiemendarm aufgenommene Wassermenge, die dem Gasaustausch und zwangsläufig auch dem Nahrungserwerb dient, setzt sich daher aus den Volumina zusammen, die a) auf den kontinuierlichen Wasserstrom in den Intervallen zwischen den Kontraktionen und b) auf die diskontinuierliche Wassererneuerung im Anschluß an die spontanen rhythmischen Kontraktionen zurückzuführen sind. Die folgende Darstellung des Mechanismus des Nahrungserwerbs bezieht sich zunächst nur auf den kontinuierlichen Wasserstrom, der durch den Wimperschlag der Kiemen- 
spalten erzeugt wird. Auf die Bedeutung der spontanen rhythmischen Kontraktionen für den Nahrungserwerb wird später eingegangen.

Die Filtration des Atemwasserstromes ist der zweite eigentliche Grundvorgang des Nahrungserwerbs. Die im umgebenden Wasser zufällig enthaltenen und mit dem Atemwasserstrom herangeführten brauchbaren und unbrauchbaren Bestandteile der verschiedensten Art und Größe, lebende und tote Planktonorganismen, organischer Detritus wie auch anorganische Sedimente, werden auf dem beschriebenen Weg des Wasserstromes durch den Kiemendarm durch zwei Filtrationsvorgänge verschiedener Art aufgefangen. Eine erste unechte Filtration wird bekanntlich durch die Mundtentakel bewirkt; diese finden sich bei den verschiedenen Familien in verschiedener Zahl, Größe und Ausbildung und können eine sehr feingefiederte und reich verzweigte Form haben. Ihre Funktion ist, größere Partikel aus dem Atemwasserstrom auszusondern, die durch Verschluß des Egestionssiphos und ruckartige Kontraktionen des gesamten Kiemendarmes sofort wieder aus dem Ingestionssipho ausgeschleudert werden. Die nervöse Grundlage dafür ist durch die Existenz der gekreuzten Reflexe gegeben (Hecri 1918, vgl. Huds 1933, 1937, van Weel 1940, Florey 1951, v. Buddenbrock 1953).

Die Rolle der Mundtentakel ist mit der Reizaufnahme und der Reusenfunktion offenbar nicht erschöpft. Gelegentlich konnte bei Clavelina und Ciona beobachtet werden, daß von den Tentakeln Schleimfäden in den Kiemendarm hineinhingen, die nur von den Mundtentakeln selbst erzeugt sein konnten. Diese Schleimfäden konnten dann auch feinere Partikel auffangen, die beim Vorbeistreichen zufällig an ihnen hängenblieben. Die Fäden rissen schließlich $a b$ und wurden in den Kiemendarm eingestrudelt, wo sie vom eigentlichen Filtrationsvorgang erfaßt wurden. Durch weitere Beobachtungen an verschiedenen Arten muß geprüft werden, ob es sich hierbei um ein regelmäßiges Verhalten handelt, oder ob es mehr zufällig auftritt und dann vielleicht durch besondere Reize ausgelöst wird.

Die feineren Partikel, die die Reuse der Mundtentakel passiert haben, gelangen in den Kiemendarm und werden beim zweiten echten Filtrationsvorgang vor dem Durchtritt durch die Kiemenspalten von feinen Schleimgeweben aufgefangen (MacGinitie 1939b), die das eigentliche Filter darstellen, das entsprechend als Kiemenschleimfilter (WERNER 1951) zu bezeichnen ist und nach dem Prinzip des endlosen Filters (WERNER 1953a) arbeitet. Dieses Schleimfilter bedeckt während des Nahrungserwerbs ständig die gesamte Innenfläche des Kiemendarmes und hat somit die gleiche Form wie dieser, ist also trichter- oder sackförmig gestaltet. Seiner Entstehung und Funktion nach setzt sich das Kiemenschleimfilter der Länge nach aus zwei getrennten Hälften zusammen; sie sind in den Anfangsphasen funktionell unabhängig voneinander und sind dem Bau des Kiemendarms entsprechend, der durch die Dorsalfalte und den Endostyl in zwei Hälften geteilt wird, als rechtes und linkes Schleimfilter zu bezeichnen. Wesentlich ist, daß das ganze Schleimfilter nicht ein starres Gebilde darstellt, das längere Zeit in Funktion ist, sondern daß es sich um ein endloses Fließband handelt, das ständig gebildet wird, ständig über die Seitenflächen der Kieme transportiert und schließlich in einem charakteristischen Sammelvorgang dauernd zu einem größeren Nahrungskörper geformt wird. Der Vorgang des Nahrungserwerbs wird also wesentlich bestimmt durch das Vorhandensein und die Wirksamkeit des Schleimfilters. Dementsprechend sind 
mehrere Teilvorgänge zu unterscheiden: a) die Bildung des Schleimfilters, b) sein Transport über die Seitenflächen der Kieme; damit ist die eigentliche Filtrationsphase gegeben; c) der Sammelvorgang, der in der Bildung eines größeren Futterkörpers besteht, d) schließlich dessen Aufnahme in den Osophagus.

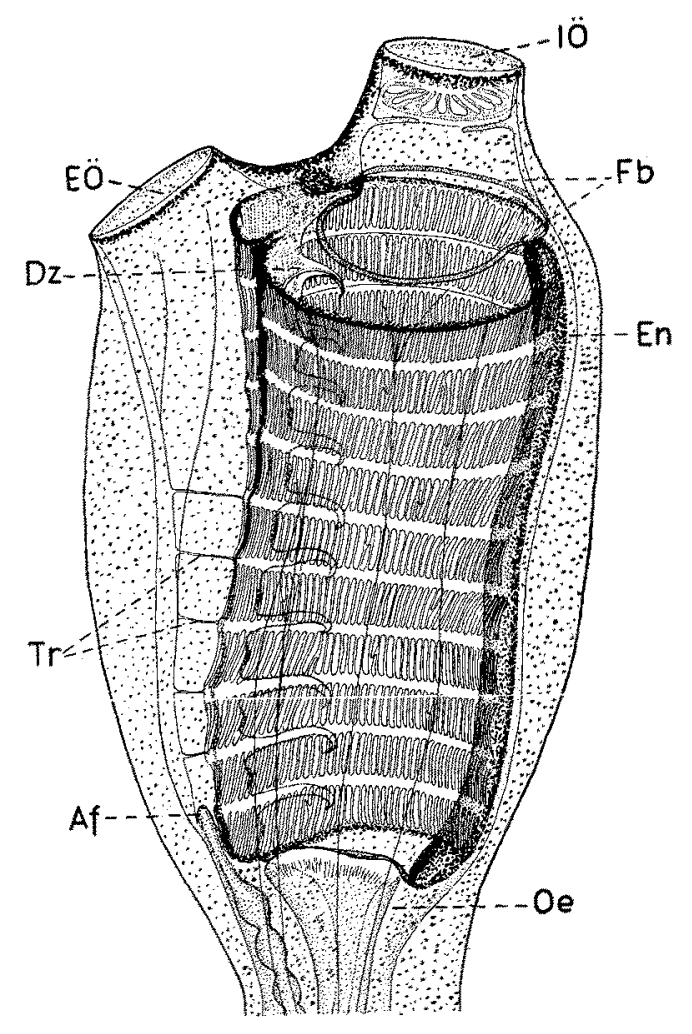

Abb. 1. Clavelina lepadiformis, Vorderkörpex, Seitenansicht. Af After, Dz Dorsalzunge, En Endostyl, EO Egestionsöffnung, Fb Flimmerbogen, IO Ingestionsöffnung, Oe Oesophagus, Tr Stütztrabekel, Freihandzeichnung.

\section{Die Bildung des Schleimfilters}

Die morphologischen Verhältnisse des Kiemendarmes sind für Clavelina in der Abb. 1 dargestellt. Die Zeichnung kann nur einen schwachen Eindruck von der Schönheit und Durchsichtigkeit dieser Art geben, die auch durch das Vorhandensein der weißgelblichen, im durchfallenden Licht schwarzen Pigmentzellen ausgezeichnet ist, die zu den charakteristischen Bändern angeordnet sind.

Es wurde schon erwähnt, daß das Schleimfilter annähernd die gleiche Form hat wie der Kiemendarm; das hängt mit seiner Beschaffenheit, seinem Bildungsort und der Art des Bildungsvorganges zusammen. Das Filter besteht aus zwei sehr feinen elastischen, normalerweise unsichtbaren Schleimgeweben, die nur mittels des Suspensionsversuches sichtbar gemacht werden können und ihre Form nur bewahren, wenn sie mit ihrer ganzen Fläche gehalten und getragen werden. 
Das Bildungsorgan ist der Endostyl, der sich auf der Ventralseite des ganzen Kiemendarmes hinzieht. Vorn schließt er sich an den Flimmerbogen (das Peripharyngealband) an, hinten an das Retropharyngealband; dieses letztere Organ kann allerdings wie in unserem Falle bei Clavelina fehlen (s. u. S. 69 f.) Der Endostyl, meist ein sehr voluminöses Organ, stellt keine eigentliche Rinne, sondern eine erhabene, weit in den Raum des Kiemendarmes vorspringende Leiste dar, die eine mehr oder weniger tiefe, rinnenförmige Einsenkung trägt. Die äußeren bzw. oberen Ränder dieser Rinne können einander sehr genähert sein. Das trifft z. B. für Clavelina und Ciona zu, wo die Rinne beim lebenden Tier in ganzer Länge nahezu geschlossen erscheint.

Der anatomisch-histologische Aufbau des Endostyls ist schon oft untersucht worden, zuletzt vergleichend von SoKóLSKA (1931, vgl. VAN WEEL 1940), so daß es genügt, hier kurz auf das Wesentliche hinzuweisen. Seine Bauelemente sind Drüsen- und Wimperzellen, die in bestimmter Weise angeordnet sind und dem Endostyl den charakteristischen Aufbau verleihen, als dessen besondere Kennzeichen der genau symmetrische Bau und damit die Zusammensetzung aus zwei spiegelbildlich gleichen Hälften zu nennen sind (Abb. 2). In jeder Hälfte befinden sich drei Längsstreifen von Drüsenzellen, die durch Streifen von Wimperepithel mit kurzen Gilien getrennt sind. Die beiden ventralen Drüsenstreifen

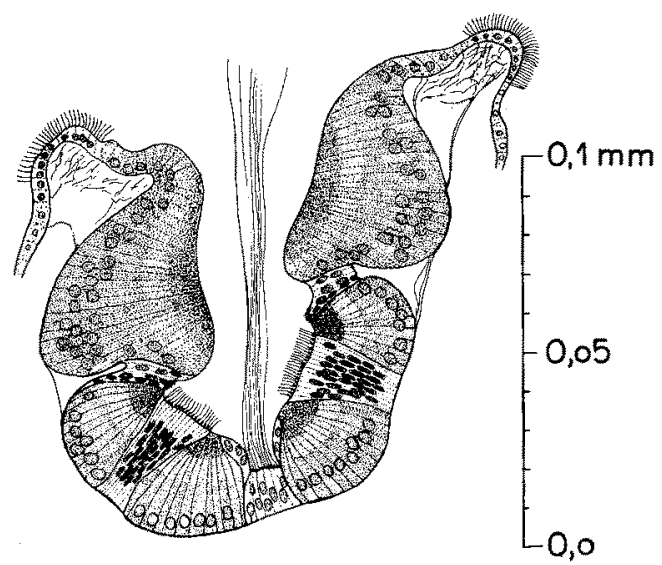

Abb. 2. Clavelina lepadiformis, Querschnitt durch den Endostyl, leicht schräg getroffen. Abbé'scher Zeichenapp. v. Leitz.

werden durch einen genau median gelegenen unpaaren Streifen von Wimperzellen getrennt, die bei Clavelina und Ciona zahlreiche dicht stehende geißelartige Cilien tragen, die durch ihre besondere Länge ausgezeichnet sind und bis zum oberen Rand der Rinne emporragen. Wichtig ist noch, daß der Endostyl in ganzer Länge von einem großen Blutgefäß unterlagert ist, dem Hypobranchialgefäß. Seine Aufgabe ist zweifellos die unmittelbare und direkte Versorgung des Endostyls mit den Stoff- und Flüssigkeitsmengen, die für die ständige Erzeugung der Schleimmengen benötigt werden.

Auf die Funktion des Endostyls und seine Arbeitsweise muß etwas näher eingegangen werden, da alle bisherigen Angaben seiner Bedeutung nicht gerecht werden. Seine allgemeine Aufgabe besteht in der Erzeugung des Schleims, worauf schon mehrfach hingewiesen wurde. Da der erzeugte Schleim unsichtbar ist, können die Arbeitsweise und die Form der erzeugten Schleimgebilde nur 
indirekt erschlossen werden, wozu als Hilfsmittel der Suspensionsversuch zur Verfügung steht ${ }^{1}$ ).

So ist es zunächst möglich festzustellen, daß aus der Rinne des Endostyls in seiner ganzen Länge ständig nach beiden Seiten hin je ein Schleimband abgegeben wird, das senkrecht zur Längsrichtung des Endostyls, also in der Querrichtung über die rechte bzw. linke Seite der Kieme, damit von der Ventralseite zur Dorsalseite transportiert wird. Jedes der beiden Schleimbänder hat daher die gleiche Länge wie der Endostyl. Beide Schleimbänder werden an der Dorsalseite in ganzer Länge bei dem später zu besprechenden Sammelvorgang vereinigt, so daß daraus die erwähnte Trichter- oder Sackform des ganzen Schleimfilters resultiert. Die Existenz und Form dieses Schleimtrichters, der die Innenseite des Kiemendarmes bedeckt, können mit Hilfe der Suspensionen bei lebenden, unbeschädigten Tieren direkt sichtbar gemacht werden. Das ist besonders eindrucksvoll bei der durchsichtigen Clavelina; bei Verwendung einer genügend dichten Suspension ist der Schleimtrichter durch die aufgefangenen Partikel minutenlang in voller Ausbildung sichtbar (s.u. Abb. 3, 4, S. 68 f.), wie es neuerdings auch JørGensen und GoldBerg (1953) für die jungen durchsichtigen Exemplare von Ciona beschrieben haben. Da die beiden Schleimbänder, aus denen sich der Schleimtrichter zusammensetzt, vom Wasser durchströmt werden, kann es sich nicht um amorphe, flächenhaft ausgebildete Schleimmassen handeln, vielmehr müssen sie aus regelrechten Geweben bestehen. Dem Endostyl ist damit die ganz bestimmte Aufgabe zugewiesen, zwei getrennte Schleimgewebe zu produzieren.

Diese Funktion erklärt zunächst einmal den spiegelbildlich gleichen Aufbau aus zwei Hälften. Es kann keinem Zweifel unterliegen, daß jede Hälfte des Endostyls eine funktionelle Einheit darstellt. Sein komplizierter Aufbau bliebe unverständlich, wenn in der Rinne nur eine einheitliche amorphe Schleimmasse produziert würde, die erst an den oberen Rändern durch die Tätigkeit der Wimpern der Wimperzellstreifen zu den beiden Schleimbändern ausgezogen würde. Vielmehr müssen wir uns die Tätigkeit des Endostyls so vorstellen, daß jede Hälfte auf der dem Lumen der Rinne zugewandten Seite je ein Schleimgewebe erzeugt. Dieses wird durch die Tätigkeit der Wimperzellen zum oberen Rand der Rinne befördert, hier auf die Außenseite des Endostyls umgeschlagen und weiter auf die Seitenflächen der Kieme transportiert.

Wie ist es aber zu erklären, daß aus der nahezu geschlossenen Rinne des Endostyls zwei getrennte Schleimgewebe austreten können, ohne daß sie stärıdig in ganzer Länge verkleben? Offenbar wird dies verhindert durch die dicht stehenden langen medianen Cilien, die eine Längstrennwand darstellen, durch die das Lumen der Rinne vom Grund bis zum oberen Rand in zwei Hälften geteilt wird. Dementsprechend können die von jeder Endostylhälfte erzeugten beiden Schleimgewebe an keiner Stelle miteinander in Berührung treten. Ihrer speziellen Funktion nach ist also die Aufgabe der langen medianen Cilien eine mehr passive. Das steht mit der Beobachtung in Ubereinstimmung, daß sie keinen schnellen und aktiven Wimperschlag zeigen. Bei lebend präparierten Tieren von Ciona, bei denen der Kiemendarm entlang der Dorsalseite geöffnet

1) Für die Untersuchungen wurden meist Suspensionen von pulverisierter Tierkohle verwendet, die wegen ihrer völigen Unschädlichkeit Suspensionen von Karmin oder Tuschelösungen vorzuziehen sind. Die Suspensionen von Kohle können allerdings jeweils nur kurze $Z$ eit verwendet werden, da sich die Kohleteilchen im Seewasser ziemlich schnell zusammenballen. 
und nach beiden Seiten umgeschlagen ist, kann durch den Suspensionsversuch gezeigt werden, daß alle mit Wimperzellen besetzten Flächen des Kiemendarms den Wimperschlag eine ganze Weile unverändert beibehalten. Die Gesamtheit der bläulich-irisierenden medianen Wimpern des Endostyls, soweit sie von außen in dessen sehr schmalem Spalt sichtbar wird, zeigt dann nur eine langsame undulierende Bewegung in der Längsrichtung des Organs, wie sie auch schon Roule (1884, vgl. ORToN 1913) gesehen hat').

Nun ist von anderen Ascidien z. B. von Molgula beschrieben, daß dem Endostylgrund die langen medianen Cilien vollständig fehlen (SokóLSKA 1931). Steht dieser abweichende Befund nicht im Gegensatz zu der hier gegebenen Deutung, die die betr. Wimpern als notwendiges, wenn auch mehr passives Funktionselement erscheinen läßt? Eine vorläufige eigene Untersuchung an Molgula tubifera Orstedt ergab, daß die längeren medianen Cilien nicht vollständig reduziert sind; vielmehr findet sich bei dieser Art eine einzelne Längsreihe von längeren Wimpern genau in der Mitte des Endostylgrundes. Die Wimpern stehen aber in jedem Fall nicht dicht genug und sind auch zu kurz, als daß sie wie bei Clavelina oder Ciona eine mediane Trennwand des Endostyllumens abgeben könnten. Im übrigen ist der Bau des Endostyls bei Molgula der gleiche, und es liegt kein Hinweis darauf vor, daß seine Funktion eine andere sein könnte. Daher muß durch eine andersartige Vorrichtung verhindert werden, daß die beiden von den Seitenflächen des Endostyls gebildeten Schleimgewebe miteinander in Berührung treten und verkleben können. Es wird vermutet, daß diese Aufgabe dadurch in sehr einfacher Weise gelöst ist, daß die beiden Hälften des Endostyls einen weiteren Abstand haben, daß der Endostyl also ein wesentlich größeres Lumen hat als bei Ciona und Clavelina, bei denen, wie erwähnt, die oberen Ränder der Rinne einander sehr genähert sind. Wenn bei der lebenden Molgula die beiden Hälften des Endostyls weiter auseinander klaffen, wird von vornherein vermieden, daß die beiden bei der Bildung von der Mitte nach den Seiten divergierenden Schleimgewebe verkleben können.

Läßt sich schließlich eine Vorstellung davon gewinnen, wie der Endostyl bei der Herstellung der Schleim ge w ebe arbeitet? Es ist zunächst darauf hinzuweisen, daß die Tätigkeit der Bauelemente, der Drüsen- und Wimperzellen, infolge der Undurchsichtigkeit und Kompaktheit des Organs, ferner wegen der Kleinheit der Bauelemente audh bei den jüngsten, noch sehr durchsichtigen Tieren einer direkten Beobachtung und Analyse bislang nicht zugänglich war. Auch die Struktur der Schleimgewebe, die durch Fixierungsflüssigkeiten, z. B. Formalin, gehärtet und dann in kleinen Stücken in einer Ebene ausgebreitet und unter dem Mikroskop betrachtet werden können, gestattet keinen direkten Hinweis auf ihre Entstehungsweise. Grundsätzlich bestehen zwei Möglichkeiten: a) die Schleimfilter sind regelrechte Gewebe, haben also eine ganz bestimmte

1) Anm. b. d. Korrektur. Bei einer ergänzenden Kontrolluntersuchung an Ciona stellte sich inzwischen heraus, daß jede einzelne der langen medianen Geißeln uberdies auch eine charakteristische aktive Form der Bewegung aufweist, die als unaufhörliche intensive schlängelnde Wellenbewegung von der Basis bis zur Spitze zu kennzeichnen ist. Bei der Gesamtheit dieser Cilien entsteht so der besondere Eindruck eines ständigen schnellen Fließens, was zweifellos die Ursache für das intensive bläuliche Irisieren ist. Auch Roule hat diese Bewegung schion beschrieben: "Les mouvements... assez lents, dirigés dans le sens de la longueur du raphé, sont comme formés par des séries d'ondulations partant des bases d'insertion" (pag. 59). Diese fließende Bewegung der medianen Geißeln hat wahrscheinlich die Bedeutung, den Transport der Schleimgewebe auf jeder Seite aus dem Grunde an die Oberfläche der Rinne zu erleichtern. 
regelmäßige Struktur, die sie einem bestimmten Formungsprozeß verdanken; b) es handelt sich um ungeformte Gewebe, die keine besondere Struktur aufweisen, deren Bestandteile vielmehr eine regellose Anordnung haben, wie sie etwa eine dünne Schicht Watte aufweist.

ad a) Es erhebt sich die Frage, ob es einen bekannten Vorgang gibt, der eine regelmäßige gewebeartige Beschaffenheit der von den Endostylhälften erzeugten Schleimfilter als möglich erscheinen läßt. U.W. ist der einzige vergleichbare, näher analysierte Vorgang die Bildung des Mantelschleimfilters bei dem Prosobranchier Crepidula fornicata (WERnER 1953 a). Das Bildungsorgan, die Mantelfilterdrüse, ist ebenfalls aus Drüsen- und Wimperzellen gebaut, die eine charakteristische Anordnung aufweisen. Aus der Anordnung und den spezifischen Tätigkeiten der Bauelemente konnte bei Crepidula die Arbeitsweise des Bildungsorgans erschlossen werden, das an seiner Oberfläche ein regelrechtes netzartiges Gewebe aus Quer- und Längsfäden erzeugt. Das sehr feine Schleimgewebe hat dementsprechend viereckige Maschen, die es in ganzer Dicke durchsetzen.

Die bisherigen Untersuchungen über den feineren Bau des Endostyls der Ascidien haben keinen eindeutigen Hinweis auf eine vergleichbare Arbeitsweise erkennen lassen. Uber die bekannten Tatsachen des Aufbaues aus mehreren Längsstreifen von Drüsen- und Wimperzellen hinaus wurde noch festgestellt, daß in dem auf jeder Seite zwischen den ventralen und mittleren seitlichen Drüsenzellstreifen gelegenen Wimperzellstreifen die Wimpern zu mehreren, der Längsrichtung des Endostyls parallelen Reihen angeordnet sind, während die Anordnung der Wimpern bei den übrigen mit Wimperzellen besetzten Zonen des Endostyls eine regellose ist. Das läßt vermuten, daß die Anordnung der Wimpern nicht zufällig ist, sondern daß sie eine bestimmte Aufgabe haben, die sich nur auf die Erzeugung der Schleimgewebe beziehen kann. Aus der Ausdehnung dieses Wimperzellstreifens, der im Querschnitt (s. o. Abb. 2) aus zahlreichen, eng zusammengedrängten Zellen mit schmalen, stark färbbaren Kernen besteht, ist weiterhin zu schließen, daß er das für die Formung und den Transport der Schleimgewebe auf den Flächen des Endostyls wichtigste Organ ist. Doch reichen diese Besonderheiten nicht aus, um die Tätigkeit des Endostyls so kennzeichnen zu können, daß daraus eine geformte regelmäßige Struktur der Schleimgewebe abgeleitet werden könnte.

ad b) Daher muß mit der Möglichkeit gerechnet werden, daß anders als beim Mantelschleimfilter von Crepidula die vom Endostyl der Ascidien erzeugten Schleimgewebe ungeformt sind. SokóLsKa (1931) hat die Tätigkeit der einzelnen Drüsenzellen des Ascidienendostyls untersucht und gesehen, daß das Schleimsekret teilweise in Form von feinen Fäden und Kügelchen abgeschieden wird. Man braucht sich nur vorzustellen, daß das ständig erzeugte Schleimband infolge seiner Zusammensetzung aus den genannten Strukturbestandteilen nicht kompakt ist, sondern eine watteähnliche Beschaffenheit annimmt, um seine Filtereigenschaft ohne weiteres verstehen zu können. Diese mögliche Beschaffenheit der Schleimfilter bei den Ascidien entspricht der Auffassung, die MAcGinITIE (1945) für das Schleimnetz des Echiuriden Urechis caupo geäußert hat, wonach es eine poröse schwammartige Struktur hat, bei der die Maschen oder Poren nicht direkt von der einen zur anderen Seite hindurchgehen. Allerdings ist bei den Ascidien mit der Möglichkeit der strukturlosen Beschaffenheit der Schleimgewebe u. E. nur schwer der komplizierte Bau des Endostyls in Einklang zu bringen. Ergänzend ist noch zu sagen, daß jedenfalls die langen medianen Cilien 
des Endostylgrundes keine wesentliche Rolle bei der Bildung der Schleimfilter spielen, da sie ja bei Molgula stark reduziert sind.

Wenn sich nach allem die Funktion des Endostyls bei der Herstellung der beiden Schleimgewebe einer näheren Analyse noch entzieht, so ist doch zusammenfassend festzustellen, daß aus der Tätigkeit und Anordnung der Drüsen- und Wimperzellen als Gesamtleistung zwei getrennte Schleimgewebe resultieren. Dabei sind die Drüsenzellen die Materialproduzenten, während der gleichmäßige (oder in bestimmter Weise rhythmisch koordinierte?) Wimperschlag der Wimperzellen das dynamische Element darstellt, das das Material zu (geformten oder ungeformten) Geweben zusammenfügt und es als zwei Schleimbänder aus der Endostylrinne heraustreten läßt.

\section{Der Transportvorgang, die eigentliche Filtrationsphase}

An die Bildung der beiden Schleimgewebe schließt sich der Transportvorgang an, durch den sie vom Endostyl nach beiden Seiten hin über die Fläche des Kiemendarmes ausgebreitet werden. Es wurde schon darauf hingewiesen, daß diese Phase die eigentliche Filtrationsphase darstellt. Wesentlich ist, daß der Transport ständig erfolgen muß, solange die beiden Filter vom Endostyl gebildet werden, da sie ja nach dem Prinzip des endlosen Filters arbeiten. Durch die ständige Erneuerung wird das Verstopfen des Filters verhindert, was bei einem stationären Filter notwendig eintreten müßte. Weiterhin werden die beiden Filter erst durch den Transportvorgang in dem notwendigen Spannungszustand erhalten, der allein ihre Filterfähigkeit gewährleistet. Ohne die Aufrechterhaltung des Spannungszustandes würden sie zu amorphen Schleimmassen verkleben.

Die Bauelemente der Transportvorrichtungen sind Wimperzellen mit sehr kurzen Wimpern (s. Tab. 1), die durch den gerichteten Schlag die Schleimgewebe weiterbefördern. Nach MacGintTIF (1939b) dringen die Wimpern dabei wie feine Häkchen etwas in den Schleim ein. Die Wimperzellen sind nicht gleichmäßig über die Innenflächen des Kiemendarmes verteilt, sondern an bestimmten Stellen gehäuft, so daß man von besonderen Transportorganen sprechen muß. Sie treten entsprechend den Unterschieden in der Morphologie des Kiemendarmes bei den verschiedenen Familien der Ascidien in wechselnder Ausbildung auf.

Grundsätzlich lassen sich nach der Verteilung zwei Gruppen von Transportorganen unterscheiden: a) solche, die außerhalb der mit Kiemenspalten versehenen Flächen des Kiemendarmes, und b) solche, die im Bereich dieser Flädhen selbst liegen.

ad a) Nach dem Beginn und der Richtung des Transportes der beiden Schleimgewebe sind zuerst die Wimperbänder auf den äußeren Rändern der Endostylrinne zu nennen. Sie erfassen die aus der Rinne emporgleitenden Schleimbänder und lenken sie auf die Seitenflächen des Kiemendarmes um, da ihr Wimperschlag quer zur Längsrichtung von der Ventral- zur Dorsalseite gerichtet ist.

Ein weiteres wichtiges, allen Ascidien gemeinsames Transportorgan ist der schon erwähnte Flimmerbogen (engl. peripharyngeal groove), der in der Präbranchialzone des Kiemendarmes liegt. Wie schon die verschiedenen Bezeichnungen: Flimmerbogen, Peripharyngealband, Peripharyngealrinne erkennen lassen, wechselt die Ausbildung dieses Organs bei den verschiedenen Formen. 
Tabelle 1

Länge der Cilien an den Wimperepithelien des Kiemendarmes, nach Messungen an lebenden Tieren.

Maße in $\mu$

\begin{tabular}{|c|c|c|c|c|c|c|}
\hline \multirow[b]{2}{*}{ Spezies } & \multirow[b]{2}{*}{$\begin{array}{l}\text { Kiemen- } \\
\text { spalten }\end{array}$} & \multicolumn{2}{|c|}{ Endostyl } & \multirow[b]{2}{*}{$\begin{array}{l}\text { Flimmer- } \\
\text { bogen }\end{array}$} & \multirow[b]{2}{*}{$\begin{array}{c}\text { Kieme } \\
\text { Flimmer- } \\
\text { reifen bzw. } \\
\text { Papillen }\end{array}$} & \multirow[b]{2}{*}{$\begin{array}{c}\text { Dorsal- } \\
\text { falte bzw. } \\
\text {-zungen }\end{array}$} \\
\hline & & $\begin{array}{l}\text { medianer } \\
\text { unpaarer } \\
\text { Wimper- } \\
\text { streifen }\end{array}$ & $\begin{array}{l}\text { obere } \\
\text { Flimmer- } \\
\text { streifen }\end{array}$ & & & \\
\hline $\begin{array}{l}\text { Clavelina } \\
\text { lepadiformis }\end{array}$ & 20 & ca. 200 & 7 & $\begin{array}{c}\text { nicht } \\
\text { gemessen }\end{array}$ & 7 & 7 \\
\hline $\begin{array}{l}\text { Ciona } \\
\quad \text { intestinalis }\end{array}$ & $17-24$ & $240-300$ & $5-7$ & $5-7$ & $5-7$ & $5-7$ \\
\hline $\begin{array}{c}\text { Ascidiella } \\
\text { aspersa }\end{array}$ & 20 & ca. 220 & 7 & $\begin{array}{c}\text { nicht } \\
\text { gemessen }\end{array}$ & 7 & $7-10$ \\
\hline $\begin{array}{l}\text { Phallusia } \\
\quad \text { mammillata }\end{array}$ & 20 & ca. 270 & 7 & $"$ & $5-7$ & 7 \\
\hline \multirow[t]{2}{*}{$\begin{array}{l}\text { Molgula } \\
\text { tubifera }\end{array}$} & $17-20$ & - & $5-7$ & 5 & $5-7$ & 5 \\
\hline & $\begin{array}{l}\text { hydro- } \\
\text { motorisch }\end{array}$ & & & $\ldots \mathrm{mt}$ & motorisch & \\
\hline
\end{tabular}

Eine vergleichende Beschreibung findet sich bei SeELiger und Hartmeyer (1893-1911, Taf. 18, fig. 1-6, vgl. Huus 1937, fig. 491, 1-3).

Im Prinzip stellt der Flimmerbogen eine mit Wimperzellen besetzte, den Vorderabschnitt des Kiemendarmes ringförmig umgebende Leiste dar, deren beide Hälften ventral am Vorderende des Endostyls, dorsal unterhalb des dorsalen Flimmerorgans zusammentreffen. Das allgemeine Bauprinzip des Flimmerbogens ist in einfachster Weisc bei Botrylluis verwirklicht, wo er im Querschnitt aus einem Halbkreis von Wimperzellen besteht, die sich um ein Quergefäß legen. In ähnlicher Weise ist der Flimmerbogen auch bei Clavelina gestaltet, doch springt er hier schon sehr viel stärker in den Kiemenraum hinein vor. Die weitere Differenzierung ist dadurch gekennzeichnet, daß die wulstförmige Flimmerleiste auf der Oralseite von einer Hautfalte, der sog. Ringfalte begleitet wird, so daß sich zwischen Flimmerleiste und Ringfalte eine Rinne befindet. In der Umgebung der dorsalen Vereinigungsstelle der beiden Hälften des Flimmerbogens befindet sich bereits bei Clavelina eine Andeutung einer solchen Ringfalte, die bei Ciona in voller Ausbildung die ganze Flimmerleiste begleitet, aber selbst nicht bewimpert ist. Bei Cynthia papillosa ist auch diese Ringfalte bewimpert; der Flimmerbogen besteht hier aus zwei Flimmerfalten, was nach den bisherigen Kenntnissen als Höhepunkt der Differenzierung zu bezeichnen ist.

Welche Aufgabe hat der Flimmerbogen? Daß er beim Nahrungserwerb beteiligt ist und dem Schleimtransport dient, wird bereits von den älteren Autoren angegeben. Aber erst MacGinitre (1939 b) hat die eigentliche Aufgabe des Flimmerbogens erkannt, nämlich die Führung und Befestigung der oralen Kanten der beiden Schleimfilter. Der aus diesen gebildete Schleimtrichter ist im Kiemenraum durch den Flimmerbogen regelrecht aufgehängt. $\mathrm{Da}$ die beiden Hälften des Schleimtrichters vom Flimmerbogen gleichzeitig quer zum eindringenden Wasserstrom transportiert werden müssen, bedarf es offenbar erheblicher Kräfte, um diese beiden Aufgaben der Befestigung und des Transportes zu bewältigen. Ihnen entspricht die Ausbildung des Flimmerbogens; seine wechselnde Differenzierungshöhe ist nur im Hinblick auf seine Funktion zu deuten. Bei den Formen mit einer Ringfalte dient 
die Rinne zwischen dem eigentlichen Flimmerwulst und der Hautfalte zweifellos dazu, die vordere Kante der Schleimfilter aufzunehmen; dadurch wird ihre Führung gesichert und ein Abgleiten vom Flimmerbogen verhindert.

Dieser Funktion, der Führung und Befestigung des Schleimtrichters an seinen vorderen Rändern entspricht, daß der Flimmerbogen unmittelbar am Vorderende des Endostyls seinen Anfang nimmt, dessen obere Flimmerstreifen sich auf jeder Seite in den Flimmerbogen fortsetzen. So wird bei allen Ascidien eine direkte und einheitliche Verbindung zwischen Bildungsorgan und Vorderabschnitt des Sammelorgans, der später zu besprechenden Dorsalfalte, hergestellt. Der Cilienschlag der Wimperzellen des Flimmerbogens ist von der Ventralseite zur Dorsalseite gerichtet.

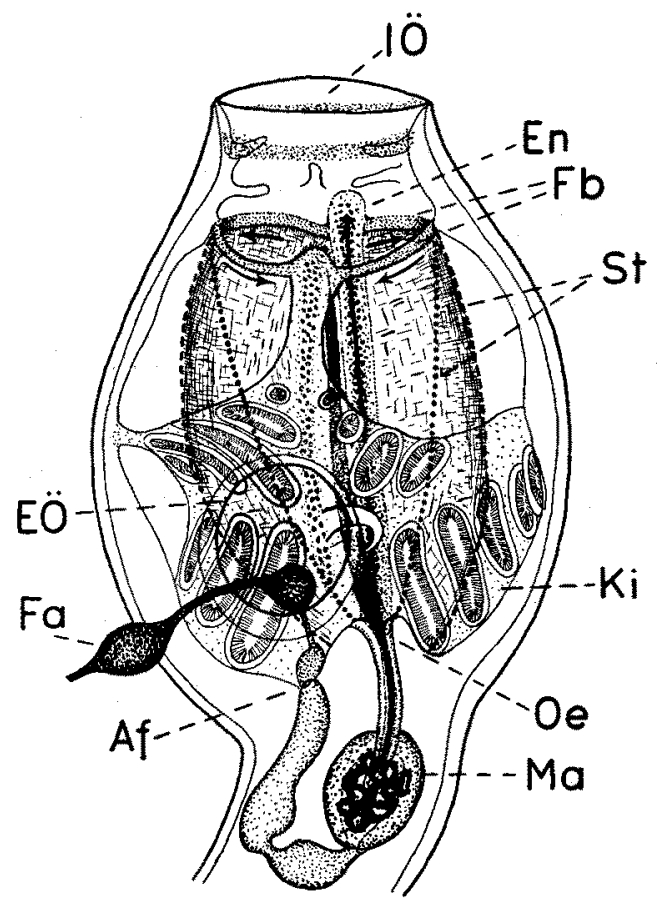

Abb. 3. Clavelina lepadiformis, Jungtier von $1,5 \mathrm{~mm}$, Dorsalansicht. Af After, En Endostyl, EO Egestionsöffnung, Fa Faeces, Fb Flimmerbogen, IO Ingestionsöffnung, Ki Kieme, Ma Magen, Oe Oesophagus, St Schleimtrichter. Durch die punktierten und gestrichelten Konturen sind zwei Phasen in der Ausbildung des Schleimtrichters dargestellt, vgl. Abb. 4 a, b.

Abbé'scher Zeichenapp, v. Leitz.

Daß dem Flimmerbogen in der geschilderten Weise tatsächlich eine ganz wesentliche Aufgabe bei der Führung und Befestigung des Schleimtrichters und damit auch bei dessen Formbildung zukommt, geht eindeutig aus den Beobachtungen an den Jungtieren von Clavelina hervor, die zunächst noch über sehr wenige Kiemenspalten verfügen. Die nach dem lebenden Tier gezeichnete Abb. 3 ist geeignet, die Situation zu illustrieren. Der wohlausgebildete Schleimtrichter schließt vorn unmittelbar an den Flimmerbogen an und erstreckt sich hinten bis in den Oesophagus hinein. Er nimmt einen erheblich größeren Raum als dic Kieme ein, die nur zwei Reihen von Spalten aufweist. Der Schleimtrichter hängt mit seiner ganzen vorderen Hälfte frei im Raum des Kiemendarmes, ohne Verbindung mit der spaltentragenden Kiemenfläche. 
Das beweist, daß hier der Schleimtrichter fast ausschließlich vom Flimmerbogen gehalten und geführt wird (vgl. die photogr. Abb. 4).

Allem Anschein nach kommt daher bei den Jungtieren dem Flimmerbogen eine größere Bedeutung für die Bildung und Erhaltung des Schleimtrichters, damit auch für dessen Filtrationsfähigkeit zu als der Kieme, die bei den erwachsenen Tieren später die Hauptaufgabe bei der Formgestaltung und dem Transport des Schleimtrichters übernimmt. Das ist deswegen von Interesse, weil bei den Copelata und Thaliacea der Flimmerbogen in prinzipiell übereinstimmender Weise ausgebildet ist. Den Abbildungen und Angaben von Fol (1872, 1876), Fedele (1933) und Carlisle (1950) ist zu entnehmen, daß auch bei diesen Gruppen der Flimmerbogen die wesentliche Aufgabe der Führung und Befestigung, sowie auch der Formbildung des Schleimtrichters hat; daher ist auch in dieser speziellen funktionellen Beziehung ein Hinweis auf die phylogenetische Reihe erkennbar (vgl. u. S. 83 f.).

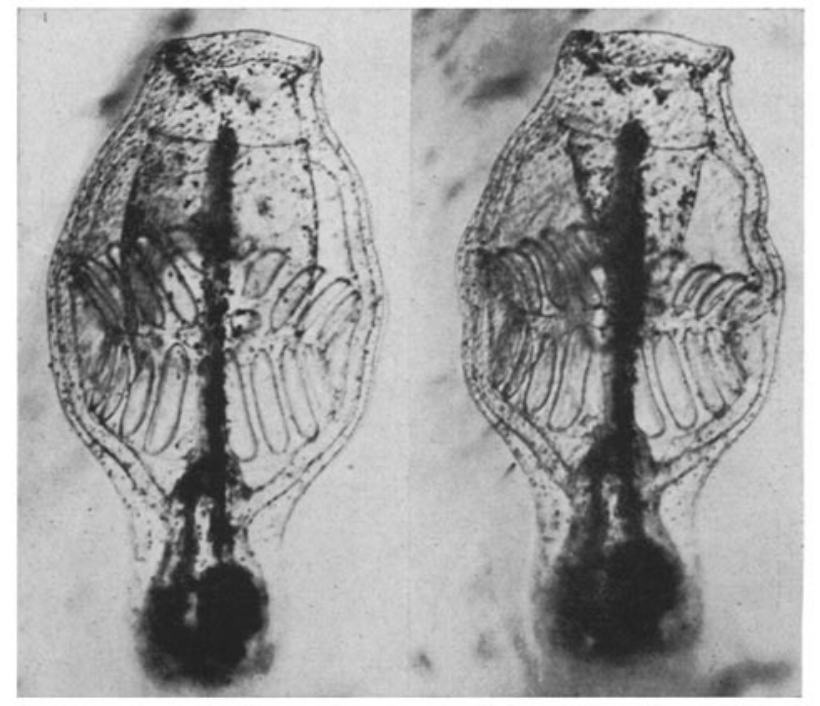

Abb. 4 a, b. Clavelina lepadiformis, Jungtier, Dorsalansicht. Zwei Phasen des Schleimtrichters, der durch die Partikel einer Kohlesuspension sichtbar gemacht ist. Vgl. Abb. 3. Umrisse teilweise leicht nachgezeichnet.

Bei den meisten Ascidien findet sich am Hinterende des Kiemendarmes ein Gegenstück zum Flimmerbogen, die sog. Retropharyngealrinne. Es handelt sich um eine mit Wimperzellen besetzte Rinne bzw. ein Band, das eine direkte Verbindung zwischen dem Hinterende des Endostyls und dem Trichter des Oesophagus herstellt und in das sich die oberen Flimmerstreifen des Endostyls fortsetzen. Zum Unterschied vom Flimmerbogen ist die Retropharyngealrinne nicht ringförmig ausgebildet; sie tritt im übrigen bei den verschiedenen Familien in etwas wechselnder Form auf. Ihre Funktion ist die Führung der hinteren "Webkanten" der beiden Schleimfilter vom Endostyl zum Oesophagus. Durch seine unpaare Ausbildung entspricht dieses Wimperband offenbar der unteren bzw. hinteren "Naht" des sackförmig gestalteten Schleimfilters. Das Vorhandensein des Retropharyngealbandes ist allem Anschein nach nicht so konstant wie das des Flimmerbogens, da, wie bereits erwähnt, ein eigentliches Retropharyngealband bei Clavelina fehlt. Hier grenzt das Hinterende des 
Endostyls direkt an den Oesophagus an, und die oberen Flimmerstreifen des Endostyls gehen unmittelbar in die bewimperten Ränder des weiten Oesophagustrichters über. Dementsprechend ist das Hinterende des Schleimtrichters bei Clavelina nicht sackförmig, sondern ausgesprochen trichterförmig gestaltet (Abb. 5).

Nach Seeliger und Hartmeyer (1893-1911) soll auch Clavelina ein wenn auch nur kurzes und tiefes Retropharyngealband haben. Diese Auffassung trifft aber nur dann zu, wenn man den weiten Trichter des Oesophagus als nicht zu diesem gehörig betrachtet, wozu aber $u$. E. keine Veranlassung vorliegt.

ad b) Die beiden Schleimgewebe des Filters gleiten fließbandartig über die Flächen des Kiemendarmes; daher müssen sich auch im Bereich der mit Spalten besetzten Flächen Transportorgane befinden. Bekannt ist die bei den

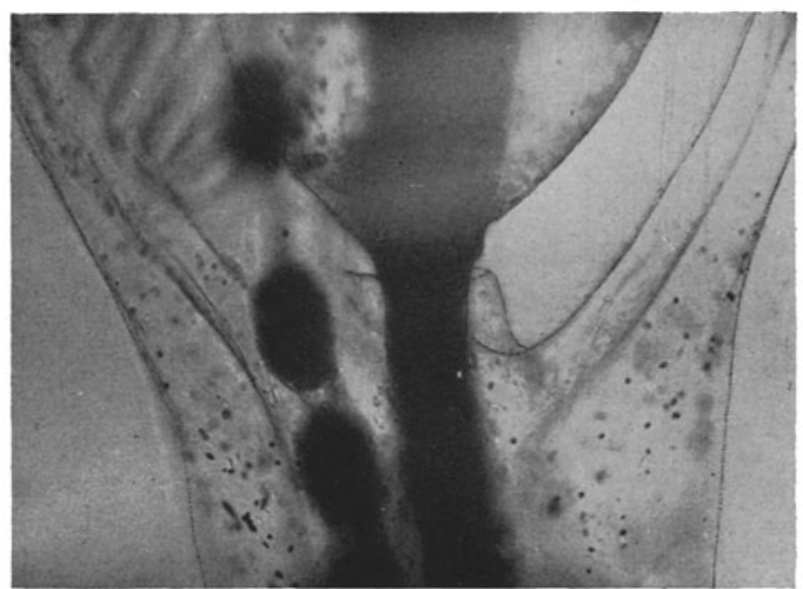

Abb. 5. Clavelina lepadiformis, Hinterabschnitt des Kiemendarmes mit dem Hinterende des Schleimtrichters und der Futterwurst, die in den Oesophagus aufgenommen wird. Links vom Oesophagus die perlschnurartige Kotschnur im Enddarm. Umrisse teilweise leicht nachgezeichnet.

verschiedenen Familien der Ascidien mannigfache Ausgestaltung des Kiemendarmes, der im einfachsten Fall und bei Jungtieren aus einfachen Querreihen von Spalten besteht, bei den höher differenzierten Formen aber einen komplizierten Bau aus Falten aufweist, die vielfach ein Gitterwerk aus Quer- und Längsfalten bilden und Gefäße genannt werden, da sie Blutbahnen enthalten. Dementsprechend sind auch die Vorrichtungen, die den Transport der Schleimgewebe über die Kieme bewirken, bei den einzelnen Gruppen verschieden ausgebildet. Als Beispiele für einfachere und für kompliziertere Verhältnisse soll hier der Transportvorgang bei Clavelina und Ciona beschrieben werden.

Die Kieme besteht bei Clavelina aus einer wechselnden Anzahl von Querreihen von Kiemenspalten (Abb. 1). Im unmittelbaren Bereich der schmalen, längsgestellten Kiemenspalten selbst befinden sich keine Vorrichtungen zum Transport der über sie hinweggleitenden Schleimfilter, vielmehr sind sie ausschließlich auf den Raum zwischen zwei Querreihen von Kiemenspalten beschränkt. Hier liegen auf der Innenseite des Kiemendarmes die sog. Flimmerreifen (SEELIGER), wohlentwickelte, in den Raum des Kiemendarms vorspringende schmale Hautfalten, die an ihrem freien Saum einen dichten Besatz von Wimperzellen tragen. Durch diese Flimmerreifen wird die ganze innere Oberfläche des Kiemendarmes in eine Anzahl von nahezu ringförmigen horizontalen 
Arkaden aufgegliedert, zwischen denen je eine Reihe von Kiemenspalten liegt. Am vorderen und hinteren Ende des Kiemendarmes befinden sich die Bildungszonen der Kiemenspalten. Von der Dorsalseite ausgehend, wachsen bekanntlich zwischen die vorderste und hinterste Reihe von Kiemenspalten Epitheibrücken ein. Dieser Vorgang setzt sich allmählich bis an die Ventralseite fort, so daß auf

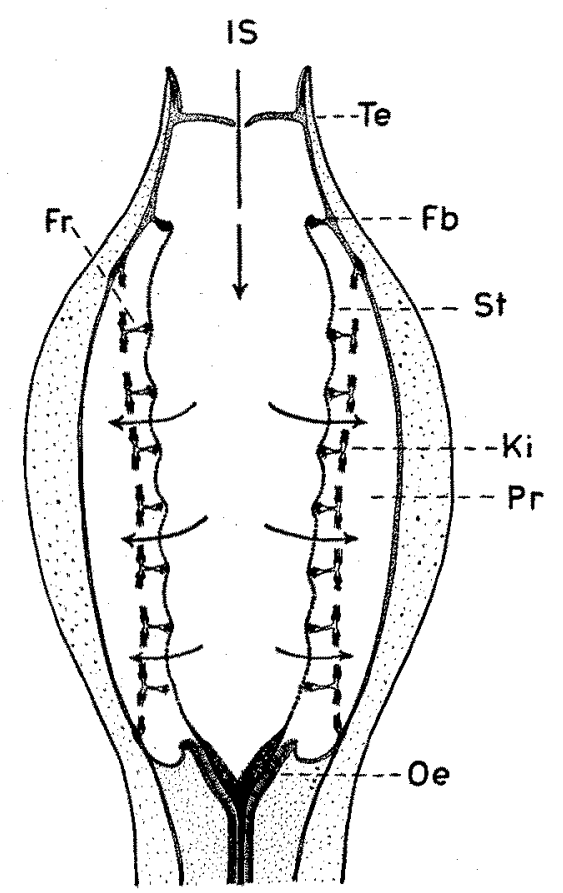

Abb. 6. Clavelina lepadiformis, schematischer. Frontalschnitt durch den Kiemendarm zur Darstellung des Filtrationsvorganges. $\mathrm{Fb}$ Flimmerbogen, Fr Flimmerreifen, IS Ingestionsstrom, Ki Kieme, Oe Oesophagus, Pr Peribranchialraum, St Schleimtrichter, Te Tentakel. Durch die Pfeile ist die Richtung des Wasserstromes kenntlich gemacht.

diese Weise eine neue Querreihe von Kiemenspalten und ebenso ein neuer Flimmerreifen entstehen (s. u. Abb. 11). Daher entspricht die Anzahl der Flimmerreifen der Anzahl von Querreihen von Kiemenspalten. Wenn man bei einem ausgewachsenen Tier, bei dem keine Kiemenspalten mehr gebildet werden, die Anzahl der Querreihen von Spalten mit $\mathrm{n}$ bezeichnet, so ist die Anzahl der Flimmerreifen $n-1$, da sich vor der ersten und hinter der letzten Querreihe von Kiemenspalten kein Flimmerreifen befindet. Auf der Ventralseite setzen die Flimmerreifen an der Basis des Endostyls an; sie haben hier eine geringere Höhe und verlaufen von hier als einheitliche Wimperbahnen bis zur Dorsalseite.

Der Suspensionsversuch zeigt bei der durchsichtigen Clavelina, daß es tatsächlich die Flimmerreifen sind, die durch den dorsalgerichteten Wimperschlag den Transport der beiden Schleimgewebe über die Kieme bewirken (s, u. Abb. 9). Durch den Druck des Wasserstromes, der die Schleimfilter durchströmt, werden sie auf die Flimmerreifen gepreßt, so daß deren Wimpern in Funktion treten können. In den Zwischenräumen zwischen den Flimmerreifen hängen dabei die zarten elastischen Schleimgewebe etwas durch, wie es im schematischen Längsschnitt Abb. 6 angedeutet ist. Die vorspringenden Falten 
der Flimmerreifen verhindern aber, daß die Schleimgewebe in den unmittelbaren Bereich der Kiemenspalten gelangen. Ergänzend ist zu erwähnen, daß die Flimmerreifen Blutgefäße enthalten.

Liegen hinsichtlich des Transportvorganges bei Clavelina einfache Verhältnisse vor, so zeigt Ciona mit dem komplizierten Bau des Kiemendarmes auf den ersten Blick verwickeltere Verhältnisse, die sich indes bei näherer Untersuchung doch auch als recht einfach herausstellen. Bei Ciona befinden sich bekanntlich auf der Innenseite des Kiemendarmes an den Kreuzungsstellen der Quer- und Längsgefäße die sog. Kiemenpapillen, die eine ganz charakteristische Form und Bewimperung haben (Abb. 7, 8). Der Suspensionsversuch, der entweder bei den jungen durchsichtigen, unversehrten oder bei den älteren, lebend präparierten Tieren das Schleimgewebe sichtbar macht, zeigt eindeutig, daß diese über die innere Oberfläche der Kieme vorspringenden Papillen die eigentlichen Transportorgane im Bereich der Kieme sind, wie schon Roule (1884) und Orton (1913) erkannt haben. Der Funktion der Kiemenpapillen entspricht ihre Form und Haltung: sie sind sämtlich zur Dorsalseite hin geneigt und

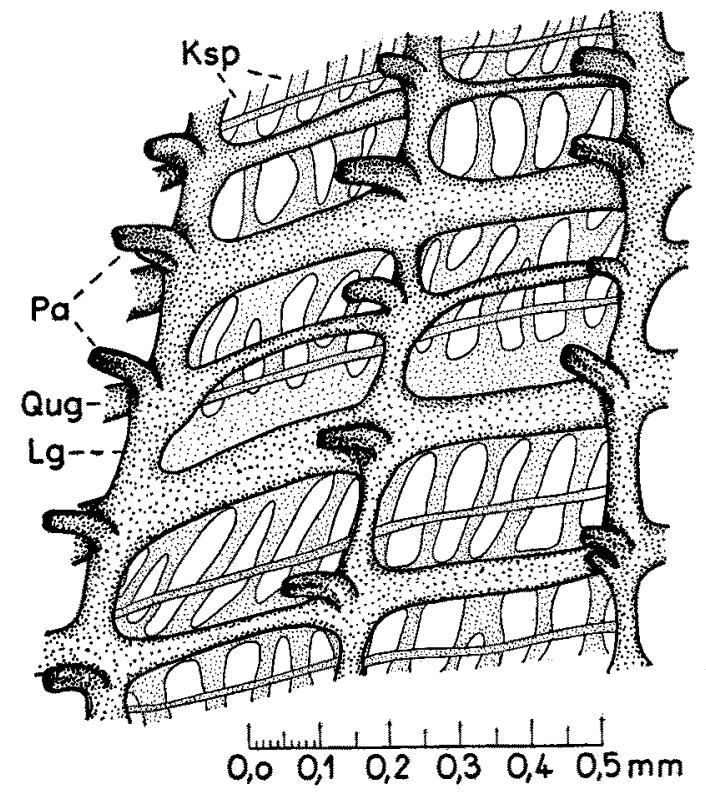

Abb. 7. Ciona intestinalis. Teil des Kiemendarmes, Aufsicht auf die Innenseite. Ksp Kiemenspalten, Lg Längsgefäß, Pa Papillen, Qug Quergefäß. Wimpern der Kiemenspalten nicht eingezeidnet. Abbé'scher Zeichenapp. v. Leitz.

tragen nur auf der äußeren, d. h. der konvexen, dem Innenraum des Kiemen darmes zugewandten Seite Wimpern (vgl. Roule 1884, Pl. 3, fig. 22, 25). Die Papillen sind in der Nähe des Endostyls am kleinsten und nehmen in Richtung auf die Dorsalseite an Größe zu. Ebenso sind die Papillen im Vorderabschnitt der Kieme etwas kleiner als im Hinterabschnitt. Durch diese Papillen, die die Schleimgewebe tragen und mit ihrem zur Dorsalseite gerichteten Wimperschlag transportieren, wird vermieden, daß diese Gewebe in den unmittelbaren Bereich der Kiemenspalten gelangen. Allerdings bängen die Schleimgewebe infolge des Wasserdruckes zwischen den im Viereck stehenden Papillen 
etwas durch, zeigen also beim Transport über die Kieme eine ausgesprochen wellige Bewegung.

Die wesentliche Aufgabe der Kiemenpapillen ist daher nicht die Vergrößerung der Oberfläche des Kiemendarmes, wenn dieser Effekt durch ihr Vorhandensein zweifellos auch erreicht wird. Ihre Aufgabe läßt sich auch aus

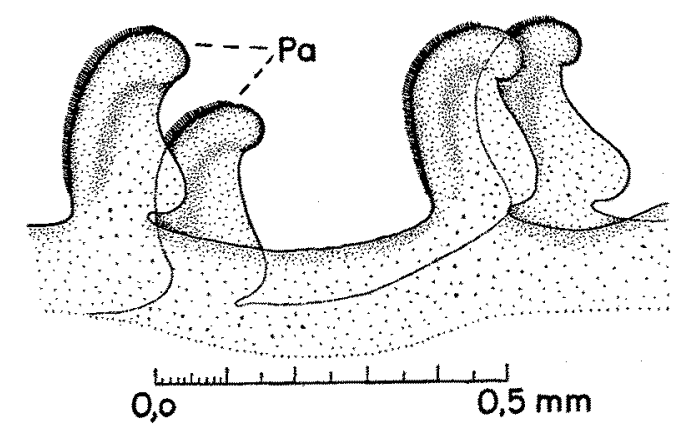

Abb. 8. Ciona intestinalis, Kiemenpapillen in der Seitenansicht. Pa Papillen. Abbé'scher Zeichenapp. v. Leitz.

ihrer Haltung unmittelbar ablesen. Wenn man nämlich bei einer durch einen Längsschnitt geöffneten und aufgespannten Ciona auf die innere Oberfläche der Kieme sieht, so erkennt man, daß die Papillen nicht genau quer zur Längsrichtung gestellt sind, sondern unter einem bestimmten Winkel schräg nach hinten und dorsal. Dieser Winkel ist bei den hinteren Papillen etwas größer als bei den vorderen. Damit ist der Weg, den das Schleimfilter bei Ciona über die Flächen des Kiemendarmes nimmt, bereits in der Haltung der Kiemenpapillen vorgezeichnet.

Der Weg der Schleimgewebe von der Ventralseite zur Dorsalseite hat nämlich im Kiemendarm nicht überall die gleiche Richtung, wie sich an den aufgefangenen Suspensionspartikeln ablesen läßt. Im vorderen Abschnitt verläuft er genau senkrecht zur Längsachse, d. h. parallel zum Flimmerbogen. Die Ursache dafür ist die Tätigkeit dieses Organs, das die wirksame Transportvorrichtung für den vordersten Abschnitt des Schleimfilters darstellt. Im mittleren und hinteren Gebiet nimmt das Schleimfilter dagegen in zunehmendem Maße einen schräg nach hinten und dorsal gerichteten Weg. Die über die beiden Seitenflächen des Kiemendarmes geführten Schleimgewebe werden hier also nicht mehr genau senkrecht zur Längsachse transportiert, sondern schräg nach hinten, weil im hinteren Abschnitt der vom Oesophagus ausgehende Zug (s. u. S. 80) stärker auf das Schleimfilter einwirkt. Der schräge Weg eines einzelnen vom Schleimfilter aufgefangenen Nahrungspartikels zeigt also die Resultierende der beiden im mittleren und hinteren Abschnitt einwirkenden Kräfte an, die bei Clavelina vom quergerichteten Wimperschlag der Flimmerreifen und vom längsgerichteten Zug des Oesophagus ausgehen (vgl. OrToN 1913). Bei dieser Form ist der schräge Weg des Schleimfilters tatsächlich nur als Resultierende der beiden Kräfte zu erklären. Bei Ciona, wo der schräg nach hinten und dorsal gerichtete Weg des Schleimfilters ebenfalls mit aller Deutlichkeit beobachtet werden kann, wird er in anderer Weise von vornherein auch bereits primär durch die Haltung der Kiemenpapillen mitbestimmt, was durch den Zug des Oesophagus noch verstärkt wird. 
VAN WEEL (1940) hat für Hungertiere von Clavelina eine von der hier charakterisierten stark abweichende Richtung des Transportweges beschrieben, die indes nach unseren Beobachtungen nicht dem normalen Verhalten entspricht. Obrigens stimmt seine Textbeschreibung pag. 53 mit der in seiner Abbildung fig. 1 a durch Pfeile skizzierten (in jedem Fall unzutreffenden) Transportrichtung nicht überein.

Hecht (1918, vgl. Berrill 1950) hat für die Funktion der Kiemenpapillen eine andere Deutung gegeben. Bei Phallusia nigra Savigny (syn. Ascidia atra Lesueur) sollen durch lokal begrenzte Kontraktionswellen jeweils zwei Reihen von Kiemenpapillen in Berührung gebracht werden, so daß auf diese Weise die "Schleimfäden" über die Fläche des Kiemendarmes befördert werden sollen. $\mathrm{Ob}$ in der von Hecht angegebenen Weise bei anderen Ascidien Wellen von Muskelkontraktionen beim Transport der Schleimfilter beteiligt sind, bedarf der Nachprüfung. Bei Ciona konnten derartige in regelmäßiger Weise über den Kiemendarm verlaufende Kontraktionswellen nicht beobachtet werden, so daß bei dieser Art der Schleimtransport über die Flächen des Kiemendarmes allein durch den Wimperschlag der Kiemenpapillen bewirkt wird.

Infolge des Transportvorganges ist die gesamte Innenfläche des Kiemendarmes vom Flimmerbogen bis zum Retropharyngealband und Oesophagus ständig von dem Schleimfilter bedeckt. Das gilt also auch für den Vorderabschnitt des Kiemendarmes, wo bei manchen Ascidien durch besondere Spalten eine direkte Verbindung mit dem Atrialraum hergestellt ist (vgl. HoYLe 1953, pag. 553). Daher findet der Wasserstrom während des Filtrationsvorganges keine Stelle, die nicht die Schleimbedeckung trüge. So erklärt sich, daß ausschließlich das rechte und linke Schleimgewebe das eigentliche Filter darstellen, das der Wasserstrom passieren muß, bevor er die Kiemenspalten oder die erwähnten anderen Spalten erreicht. Dabei müssen notwendig alle Suspensionsbestandteile aufgefangen werden, die nicht klein genug sind, um die Maschen oder Poren der feinen Gewebe passieren zu können. Die Kieme spielt für das Schleimfilter nur die Rolle des Stütz- und Transportorgans. So werden auch von den Wimpern der Transportorgane nicht die aufgefangenen Partikel befördert, sondern nur die Schleimgewebe, die ihrerseits als Fließbänder die Partikel mitnehmen. Damit ist gezeigt, daß der Transportvorgang die eigentliche Filtrationsphase darstellt.

Weiterhin wird klar, daß die Kiemenspalten, die in ihrer verschiedenartigen Gestaltung und Anzahl den Wänden des Kiemendarmes die Form eines Maschenwerks verleihen, am Filtrationsvorgang nicht unmittelbar beteiligt sind. Schon die Länge ihrer Wimpern deutet an, daß sie für diese Aufgabe ungeeignet sind. Das läßt sich außerdem durch einen Versuch bestätigen. Wenn nämlich die Schleimerzeugung während der Wasserstromerzeugung aussetzt (s. u. S. 85 f.), so werden die Partikel einer Suspension nicht mehr aufgefangen, vielmehr werden sie durch die Kiemenspalten in den Peribranchialraum und aus diesem durch den Egestionssipho wieder ausgestrudelt. Dabei kann häufig beobachtet werden, daß Partikel an den Wimpern der Kiemenspalten hängen bleiben. Es handelt sich aber keineswegs um einen normalen Vorgang des Nahrungserwerbs, sondern um eine durchaus unerwünschte Erscheinung, die die Wimpern in ihrer normalen Funktion behindert, da sie sich oft erst nach geraumer Zeit von den Partikeln freizumachen vermögen. Zudem ist keine Vorrichtung vorhanden, durch die die so aufgefangenen Partikel gesammelt und auf irgendeinem Wege zum Oesophagus befördert werden könnten. Die von den Wimpern der Kiemenspalten früher oder später 
wieder abgestoßenen Partikel geraten zwangsläufig in den Egestionsstrom und werden wieder ausgestrudelt.

MACGinitie (1939 b) beschreibt, daß einzelne Partikel entlang den Transportbahnen zur Dorsalseite befördent werden, auch wenn die Schleimausscheidung des Endostyls und damit die Bildung der Filtergewebe unterbrochen ist. Das trat besonders bei präparierten Tieren in Erscheinung und war verbunden mit einer Schleimausscheidung des Epithels des Flimmerbogens und der Dorsalfalte. In Übereinstimmung damit ließ sich bei unseren eigenen Versuchen beobachten, daß eine geringe Schleimsekretion stets auch auf dem Flimmerbogen und auf den Flächen des Kiemendarmes stattfindet. Das läßt sich experimentell mit Hilfe des Suspensionsversuches nachweisen, wenn man bei einem lebend präparierten Tier den Endostyl in ganzer Länge vom Kiemendarm abtrennt. In Úbereinstimmung damit steht die Angabe von SeEliger und Hartmeyer (1893-1911), daß im Epithel des Flimmerbogens und der Dorsalfalte Schleimdrüsenzellen in geringer Zahl liegen, was in gleicher Weise auch für die übrigen Transportorgane des Kiemendarms zutreffen dürfte. Ihre Funktion ist sehr wahrscheinlich, durch eine ständige geringe Sekretion das Gleiten der vom Endostyl erzeugten Schleimgewebe zu erleichtern. Diese Deutung ist $u$. E. der Auffassung von MAcGrnitre vorzuziehen, daß die Schleimbildung auf dem Flimmerbogen und an der Dorsalseite nur eine anormale Erscheinung sei. Vergleichsweise sei erwähnt, daß nach FEDELE (1933) auch im Flimmerbogen der Salpen Schleimdrüsenzellen vorkommen.

\section{Der Sammelvorgang}

Aus den Mitteilungen der älteren Autoren ist bekannt, daß sich beim Nahrungserwerb der Ascidien an der Dorsalseite des Kiemendarmes aus Schleim und Nahrungsbestandteilen ein langsam rotierender Faden bildet, der hier von vorn nach hinten befördert und schließlich in den Oesophagus hineingezogen wird. Weiterhin ist bekannt, daß bei diesem Sammelvorgang die Dorsalfalte von Bedeutung ist, die bei vielen Formen in charakteristischer Weise in die sog. Dorsalzungen aufgegliedert ist. Besonders Roule (1884) hat bei Ciona die Form und Funktion der Dorsalzungen beim lebenden Tier untersucht und festgestellt, daß sie sämtlich nach einer Seite, und zwar von oben, d. h. von vorn gesehen, zur rechten Seite umgebogen sind, so daß sie in ihrer Gesamtheit eine Art Kanal bilden, der den mit Nahrungspartikeln beladenen Schleimfaden aufnimmt. Mit dieser Beobachtung ist RovLe der Erkenntnis der Bedeutung der Dorsalzungen und der Art des Sammelvorganges bereits recht nahe gekommen.

Der im Kiemendarm an der Dorsalseite stattfindende Sammelvorgang ist mit Hilfe des Suspensionsversuches leicht zu beobachten. Eine nähere Prüfung ergab, daß dieser Vorgang allem Anschein nach noch von keinem Autor in seinem wirklichen Ablauf beschrieben ist. Die entscheidende Beobachtung gelang im Frühjahr $1951 \mathrm{E}$. WERNER erstmals an lebenden unversehrten Exemplaren von Ciona, die aufrecht gestellt waren, so daß man durch den Ingestionssipho in den Kiemendarm hinein und direkt auf die Dorsalseite sehen konnte. An der durchsichtigen Clavelina konnte dann später mit geringer Mühe bestätigt werden, daß sich der Sammelvorgang in genau der gleichen Weise abspielt wie bei Ciona, was ganz allgemein für alle Ascidien zutreffen dürfte. Wegen der bei Clavelina einfacheren Verhältnisse sei der Sammelvorgang wieder zu- 


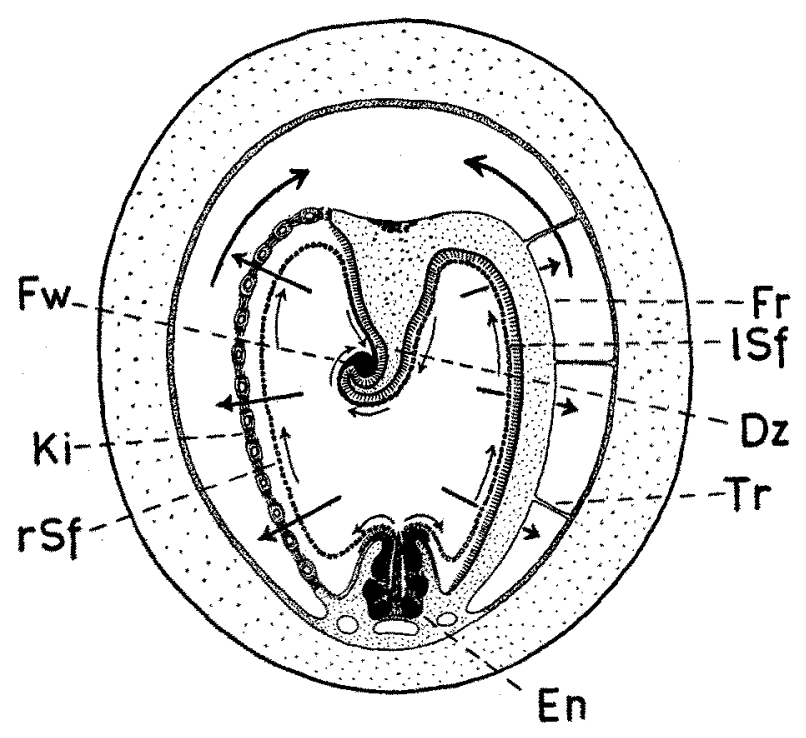

Abb. 9. Clavelina lepadiformis, kombinierter schematischer Querschnitt durch den Kiemendarm nach Seeliger und Hartmever (1893-1911, Taf. VII fig. 6, umgezeichnet) zur Darstellung des Transport- und Sammelvorganges. In der linken Bildhälfte ist der Schnitt durch eine Reihe von Kiemenspalten gelegt, in der rechten durch einen Flimmerreifen. Dz Dorsalzunge, En Endostyl, Fr Flimmerreifen, FW Futterwurst, Ki Kieme, ISf linkes, rSf rechtes Schleimfilter, Tr Stütztrabekel. Die kleinen Pfeile geben die Richtung des Transports der Schleimfilter an, die großen Pfeile die Richtung des Wasserstromes.

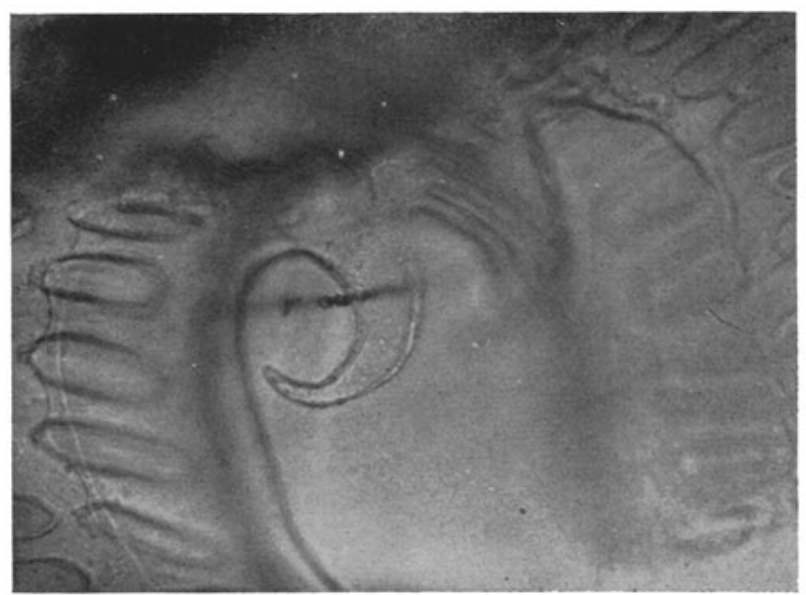

Abb. 10. Clavelina lepadiformis, Blick yon oben in den Kiemendarm und auf eine Dorsalzunge in der normalen Haltung beim Sammelvorgang. In der Krümmung der Dorsalzunge ist der Futterfaden sichtbar.

erst für diese Form beschrieben; er ist im schematischen Querschnitt Abb. 9 bildlich dargestellt.

Die Flimmerreifen auf der Innenseite des Kiemendarmes gehen bei $\mathrm{Cla}$ velina unmittelbar in die Flimmerbänder der Dorsalzungen über, die an deren seitlichem Saum bis zur Spitze verlaufen. Der Form nach handelt es sich bei den Dorsalzungen um Hautlappen, die in der Horizontalen abgeplattet sind. Bei jüngeren Tieren haben sie in gestrecktem Zustand mehr die Form eines 


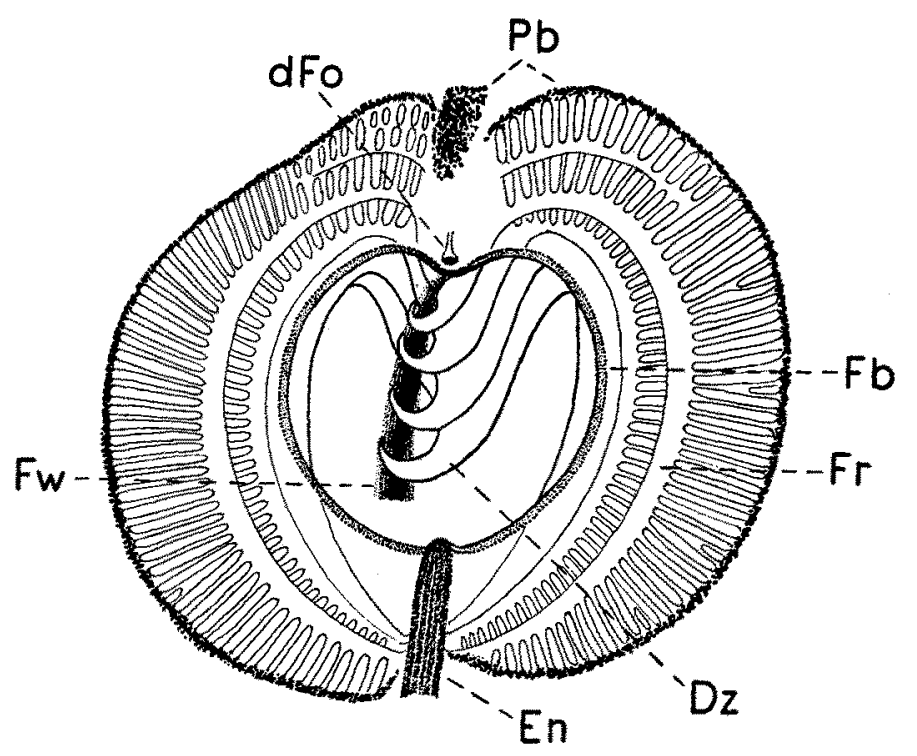

Abb. 11. Clavelina lepadiformis, Blick von oben in den Vorderabschnitt des Kiemendarmes. $\mathrm{D}_{z}$ Dorsalzunge, En Endostyl, Fb Flimmerbogen, dFo dorsales Flimmerorgan, Fr Flimmerreifen, Fw Futterwurst, Pb Pigmentbänder. Freihandzeichnung.

gleichschenkligen Dreiecks, das an der Dorsalseite mit relativ breiter Basis ansetzt. Später nehmen sie eine sehr lange und schmale Form an und ragen dann weit in den Kiemendarm vor. Die älteren Tiere lassen auch deutliche Unterschiede in der Größe der Dorsalzungen erkennen. Die kleinsten Dorsalzungen befinden sich am Vorderende des Kiemendarmes; sie nehmen bis zum Hinterende erheblich an Größe zu. Untersucht man an den Dorsalzungen die Richtung des Wimperschlages der seitlichen Wimpersäume, so sieht man, daß er auf beiden Seiten von der Basis zur Spitze führt. Daher verläuft von der Basis des Endostyls über die Flimmerreifen bis zur Spitze der Dorsalzungen ein einheitlicher dorsal gerichteter Wimperstrom. Ebenso wie an den übrigen Transportorganen haben auch die Wimperzellen an den Dorsalzungen kurze Wimpern (s. Tab. 1). Der zur Dorsalseite gerichtete Wimperstrom dieser zusammenhängenden Wimperbahn bewirkt, daß die auf beiden Seiten des Kiemendarmes transportierten Schleimgewebe notwendig an der Spitze der Dorsalzungen zusammentreffen müssen. Bei dem laufenden Nachschub würden sie zu formlosen Schleimmassen zusammengeschoben, wenn nicht durch den sinnreichen Mechanismus des Sammelvorganges ständig ein größerer, regelmäßig geformter Nahrungskörper gebildet würde.

Während des Filtrationsvorganges sind die Dorsalzungen sämtlich sichelförmig zur rechten Seite gekrümmt, so daß sie mit der konvexen Seite beinahe die Fläche des Kiemendarmes berühren (Abb. 10); sie bilden so in ihrer Gesamtheit mit ihrer konkaven Seite einen in den $Z$ wischenräumen durchbrochenen Kanal. Durch den zur Spitze gerichteten Wimperschlag entsteht auf der rechten Seite der Dorsalzungen, $d$. h. auf der der Innenseite des Kanals zugewandten konkaven Seite eine Rotationsbewegung; diese bewirkt, daß das von der rechten Seite der Kieme herangeführte Schleimgewebe in den Kanal aufgenommen und hier wie ein Teppich aufgerollt wird. Das von der linken Seite des Kiemendarmes herangeführte zweite Schleimgewebe dagegen muß 
zuerst über die obere konvexe linke Seite der Dorsalzungen hinweg befördert werden und kommt erst jetzt in den Bereich des sich primär im Kanal aufrollenden rechten Schleimfilters. Es wird von diesem mitgerissen und zusammen mit diesem zu einem einheitlichen Futterkörper aufgerollt. Der zur Spitze der Dorsalzungen gerichtete Wimperstrom und ihre Form und Haltung sind daher die wesentlichen Faktoren für das Zustandekommen dieses charakteristischen Sammelvorganges, durch den beide Schleimfilter in dem Kanal zu einem einheitlichen rotierenden Nahrungskörper geformt werden. Dieser hat je nach der Menge des Schleims und der aufgefangenen Nahrungsbestandteile Faden- oder Wurstform und nimmt in der Richtung von vorn nach hinten an Dicke zu. Der von den gekrümmten Dorsalzungen gebildete Kanal stellt das eigentliche Sammelorgan dar und kann als Futterrinne bezeichnet werden.

Dieser Sammelvorgang findet seiner Natur nach an der Dorsalseite ständig in der ganzen Länge des Kiemendarmes statt. Entsprechend der vorderen Führung und Befestigung der beiden Schleimgewebe durch die beiden Hälften des Flimmerbogens (s. o. S. 67 f.) muß an deren dorsaler Vereinigung das Vorderende der rotierenden Futterwurst entstehen, die sich von hier aus in die Futterrinne hineinerstreckt. Die Vereinigungsstelle der beiden Hälften des Flimmerbogens liegt, wie schon erwähnt, unter dem dorsalen Flimmerorgan und erweitert sich bei vielen Arten zu einem Flimmerfeld. Beim Blick von oben in den Kiemendarm hinein läßt sich bei Clavelina mit Hilfe des Suspensionsversuches die vordere Vereinigung der beiden Schleimfilter mit aller Deutlichkeit verfolgen (Abb. 11, vgl. Abb. 12).

Die Beobachtung, daß der Nahrungsfaden an der dorsalen Vereinigungsstelle des Flimmerbogens, also in unmittelbarer Nachbarschaft des dorsalen Flimmerorgans seinen Anfang nimmt, hat vermutlich zu der Auffassung früherer Autoren beigetragen, daß der Schleim von der mit dem Dorsalorgan in Verbindung stehenden Neuraldrüse geliefert und vom Dorsalorgan in den Kiemenraum hinein verteilt werde. Daß diese Vermutung unzutreffend ist, daß vielmehr der Schleim auch des Vorderendes des Nahrungsfadens vom Endostyl stammt, geht aus der Funktion des Flimmerbogens und dem $\mathrm{Me}$ chanismus des hier beschriebenen Sammelvorganges einwandfrei hervor.

Der Sammelvorgang bei Ciona stimmt mit dem von Clavelina vollständig überein (Abb. 13). Die Dorsalseite des Kiemendarmes ist bei Ciona in ganz der gleichen Weise in Dorsalzungen aufgegliedert, die allerdings bei dieser Form entsprechend ihrer Größe in größerer Zahl auftreten und eine größere Länge haben. Der Unterschied gegenüber Clavelina besteht lediglich darin, daß bei Ciona keine einheitlichen von der Ventralseite bis zur Spitze der Dorsalzungen durchlaufenden Wimperbahnen vorhanden sind, daß diese vielmehr zwischen den Papillen unterbrochen sind, was aber den in der Futterrinne ablaufenden Sammelvorgang nicht beeinflußt.

Es bleibt noch die Frage zu klären, wie bei den Ascidien der Sammelvorgang vonstatten geht, bei denen die Dorsalseite des Kiemendarmes nicht in die Dorsalzungen aufgegliedert ist, sondern die Form einer in das Lumen des Kiemendarmes vorspringenden dünnen Längsfalte hat, die ganzrandig sein kann, aber bei manchen Formen mehr oder weniger in Zipfel ausgezogen ist. Daß auch die in dieser Weise gestaltete Dorsalfalte das Sammelorgan für die beiden Schleimgewebe des Filters sein muß, geht schon aus der Transportrichtung des Flimmerbogens und der Transportbahnen auf den Flächen der Kieme und vor allem aus der tatsächlichen Lage der Futterwurst hervor. Bei 


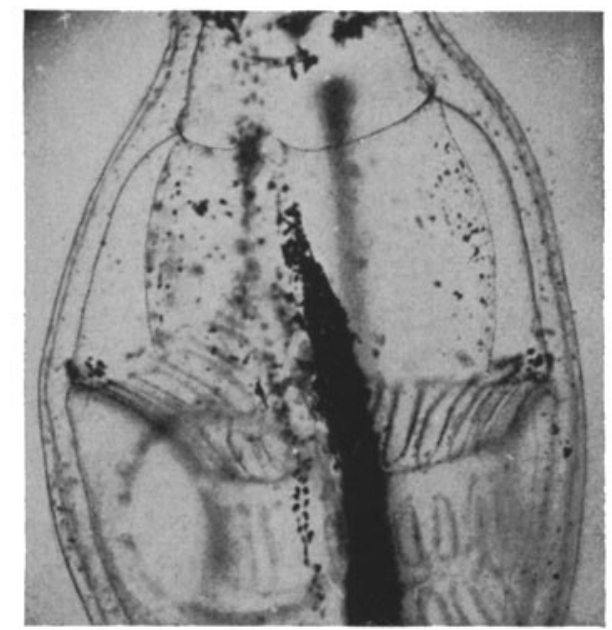

Abb. 12. Clavelina lepadiformis, Vorderabschnitt des Kiemendarmes zur Darstellung des Schleimtrichters und des Vorderendes der Futterwurst, die durch die Partikel einer Kohlesuspension sichtbar gemacht sind, vgl. Abb. 3, 4. Umrisse teilweise leicht nachgezeichnet.

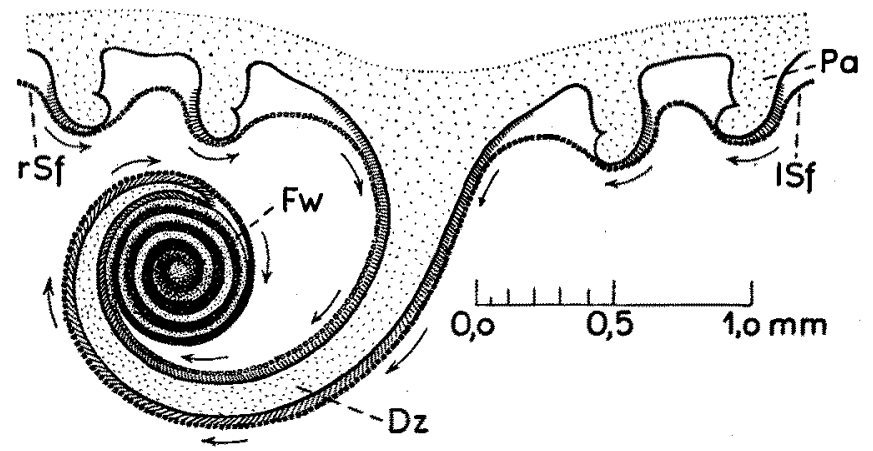

Abb. 13. Ciona intestinalis, Sammelvorgang an der Dorsalseite des Kiemendarmes, Teilquerschnitt. Dz Dorsalzunge, Fw Futterwurst, Pa Papille, ISf linkes Schleimfilter dunkel gezeichnet, ISf rechtes Schleimfilter hell gezeichnet.

den bisher untersuchten Formen Molgula tubifera Orstedt, Ascidiella aspersa O. F. Müller und Phallusia mammillata Cuvier konnte der Sammelvorgang am lebenden Tier wegen ihrer Undurchsichtigkeit nicht direkt verfolgt werden. Ebenso verhindern die zahlreichen Mundtentakel einen direkten Einblick in den Kiemendarm. Doch gelang es, den Sammelvorgang und die Funktion der Dorsalfalte beim lebend präparierten Tier zu prüfen.

Das Ergebnis ist, daß auch bei diesen mit der eigentlichen Dorsalfalte ausgestatteten Arten der Sammelvorgang in prinzipiell der gleichen Weise abläuft. Die Dorsalfalte ist in ihrer ganzen Länge zur rechten Seite umgebogen und bildet so von vorn bis hinten eine einheitliche Futterrinne; in ihrem Lumen rollt sich wie bei Clavelina und Ciona primär das rechte Schleimfilter auf, während das linke über die konvexe Seite der Dorsalfalte herübergeführt, so in die Futterrinne umgelenkt und hier mit dem rotierenden rechten Schleimfilter zusammen zu dem einheitlichen Futterkörper aufgerollt wird. In einem schematischen Querschnitt wäre also der Sammelvorgang bei diesen Formen an allen Stellen der Dorsalfalte in genau der gleichen Weise abzubilden, wie 
es bei Clavelina und Ciona nur in den horizontalen Ebenen geschehen kann, die durch die Dorsalzungen gehen.

Es ist unschwer, sich vorzustellen, daß aus der eigentlichen Dorsalfalte, wie sie Molgula, Ascidiella, Phallusia besitzen, durch Aufgliederung die Dorsalzungen entstanden sind, zumal ja die Funktion beider Bildungen völlig die gleiche ist. Von Interesse ist noch, daß sich bei Ascidiella und Molgula einerseits und Phallusia andererseits Unterschiede in der Bewimperung der Dorsalfalte finden. Bei Ascidiella und Molgula sind beide Flächen der Hautfalte völlig bewimpert; der Schlag der Wimpern ist auf beiden Seiten von der Basis zur Kante gerichtet. Bei Phallusia dagegen sind die Wimperzellen zu schmalen Wimperstreifen angeordnet, die von der Basis der Dorsalfalte zu ihrer Kante führen. Dort, wo diese Flimmerstreifen an der freien Kante endigen, ist diese zu schwach ausgebildeten Zipfeln ausgezogen. Durch diese Besonderheit ist daher bei Phallusia die Aufgliederung der Dorsalfalte bis zu einem gewissen Grade tatsächlich schon vorgezeichnet.

Berrill (1950) ist der Auffassung, daß die eigentliche Dorsalfalte aus den Dorsalzungen durch Verschmelzung hervorgegangen ist, weil sie bei wenigen und höher spezialisierten Gruppen auftritt, wie hier der Vollständigkeit halber erwähnt sei.

\section{Die Aufnahme der Futterwurst in den Oesophagus}

Wenn das Schleimfilter und die sich an der Dorsalseite bildende Futterwurst durch den Suspensionsversuch sichtbar gemacht werden, so sieht man stets, daß das hintere Ende in den Oesophagus hineinragt und in langsamer Rotationsbewegung hineingezogen wird. Die Tätigkeit des Oesophagus ist es im wesentlichen, die den Transport der in der Futterrinne rotierenden Futterwurst von vorn nach hinten bewirkt, wozu der Oesophagus durch den gerichteten Schlag seiner Wimperzellen befähigt ist, der offenbar in der Form einer Spiralbewegung herumläuft. Jedes Teilchen der Futterwurst beschreibt so während ihres Transportes den Weg einer Spirale. Man kann zuweilen beobachten, daß sich die hinteren Dorsalzungen mit ihrer Spitze nach unten bzw. hinten umbiegen, wodurch der Anschein erweckt wird, als ob sie auf diese Weise auch an dem Transport der Nahrungswurst nach hinten beteiligt sind. Doch dürfte diese Erscheinung mehr passiver Natur sein und durch den auf die Futterwurst vom Oesophagus einwirkenden $\mathrm{Zug}$ verursacht werden, der auf die feinen Spitzen der Dorsalzungen übertragen wird.

Die Tätigkeit des Oesophagus bewirkt ferner, daß das Hinterende des Schleimtrichters bei Clavelina der hinteren Kiemenfläche nicht dicht anliegt, sondern hier praktisch frei geführt und als Ganzes zusammen mit der Futterwurst in den Trichter des Oesophagus eingezogen wird (vgl. Abb. 5). Bei Ciona dagegen hat das aus den beiden Hälften zusammengesetzte Schleimfilter infolge der anderen Form des Kiemendarmes weniger die Form eines Trichters als vielmehr die eines überall anliegenden Sackes, der von seinem einen "Zipfel " aus zusammen mit der die eine "Längsnaht" kennzeichnenden Nahrungswurst in den Oesophagus hineingezogen wird.

Zum Schluß ist noch kurz zu erwähnen, daß die Nahrungswurst infolge der charakteristischen Art des Sammelvorganges als Ganzes in den Magen aufgenommen und hier zunächst regellos aufgeknäuelt wird (MACGINITIE $1939 \mathrm{~b}$ ), bis mit der fortschreitenden Verdauung eine mehr oder weniger intensive Auflösung einsetzt. 


\section{Besprechung der Ergebnisse}

1. Der Nahrungserwerb der Ascidien erweist sich bei näherer Betrachtung als ein äußerst sinnreicher Vorgang, der in der beschriebenen Form mechanisch letzten Endes auf die Tätigkeit von zwei Bauelementen, der Drüsen- und Wimperzellen, zurückzuführen ist. Es wurde versucht, diesen komplexen Vorgang mit den Hilfsmitteln der Lebendbeobachtung und des Suspensionsversuches in seine Einzelphasen aufzugliedern und auf diese Weise den mechanischen Ablauf zu erklären. Nach den Untersuchungen an Clavelina und Ciona kann der Nahrungserwerb als ein echter Filtrationsvorgang mittels eines endlosen Schleimfilters gekennzeichnet werden, der in seinem Ablauf für sämtliche Ascidien in prinzipiell übereinstimmender Weise zutreffen dürfte, und zwar in gleicher Weise für die solitären wie auch die koloniebildenden Formen, wie schon MACGINITIE (1939 b) betont hat. Das wird daraus geschlossen, daß bei aller Verschiedenheit der morphologischen Gestaltung doch in der Organisation des Kiemendarmes der gleiche Bauplan verwirklicht ist. Bei allen Formen ist das Bildungsorgan des Schleimfilters, der Endostyl, in prinzipiell und bei vielen Arten auch in den Einzelheiten tatsächlich übereinstimmender Weise ausgebildet. Daher ist anzunehmen, daß auch die Funktion überall die gleiche ist, daß also beide Endostylhälften je ein Schleimgewebe produzieren. Die beiden Schleimfilter werden anschließend über die Seitenflächen des Kiemendarmes geführt und ergeben zusammen ein einheitliches sack- oder trichtertormiges Schleimfilter, da die beiden Hälften in einem typischen Sammelvorgang ständig vereinigt werden. Durch die wechselnde Ausgestaltung des Kiemendarmes weisen die morphologischen Grundlagen des Transportvorganges, der die eigentliche Filtrationsphase darstellt, Verschiedenheiten auf, wie schon ein Vergleich der Flimmerreifen, der einheitlichen Transportbahnen bei Clavelina, und der zahlreichen getrennten Kiemenpapillen bei Ciona erkennen läßt. Die vordere Führung und Befestigung des Schleimtrichters mittels des Flimmerbogens ist aber wieder bei allen Formen die gleiche, wenn auch der Flimmerbogen in seiner Morphologie Verschiedenheiten aufweist, die nur in Richtung auf eine immer vollkommenere und wirksamere Funktion zu deuten sind. Entsprechendes gilt für die hintere Führung des Schleimfilters in dem Retropharyngealband, das als gesondertes Organ wohl nur bei den Formen fehlt, bei denen das Hinterende des Endostyls unmittelbar an den Trichter des Oesophagus grenzt. Schließlich erfolgt bei aller morphologischen Verschiedenheit der Dorsalfalte, die als einheitliche Hautfalte ausgebildet oder in einzelne Zungen aufgelöst ist, der Sammelvorgang in der von ihr gebildeten Futterrinne in übereinstimmender Weise.

An dieser Stelle muß auf die schon erwähnten Arbeiten von Hoyle (1952, 1953 , s. o. S. 58 u. 59 f.) eingegangen werden, nach denen die bislang gegebene Darstellung des Nahrungserwerbs für einige Ascidienarten durch die Einbeziehung der Wirksamkeit der spontanen rhythmischen Kontraktionen (im folgenden kurz srK genannt) erweitert werden muß. HECHT (1918) hat dieses Phänomen entdeckt, hat ihm aber keine Bedeutung zugemessen, sondern geglaubt, in ihm nur ein zwar phylogenetisch interessantes, aber funktionsloses Homologon zu den rhythmischen Muskelkontraktionen der Salpen sehen zu können. Indessen hat HoYLe mit Recht darauf hingewiesen, daß die srK des Ascidienkörpers in jedem Fall eine regelmäßige Erneuerung des Wassers im Kiemendarm bewirken und damit auch für den Gasaustausch und für den

6 Meeresuntersudungen Bd. V. H. 1 
Nahrungserwerb von Bedeutung sein müssen. Das Zeitintervall beträgt nach Hecht (1918) für Phallusia nigra durchschnittlich 5 Min., nach Yamagvchr (1931) für Styela clava 2-7 Min., nach HoxLe für Phallusia mammillata 6-9 Min. In diesen Zeitabständen wechselt also eine Periode des gleichmäßigen kontinuierlichen, durch den Wimperschlag der Kiemenspalten erzeugten Wasserstromes mit dem plötzlichen, durch Muskelkontraktionen hervorgerufenen Ausstoßen und der Erneuerung eines Teiles des im Körper befindlichen Wasservolumens in regelmäßiger Weise ab. Nach Hoyle ist der Anteil der Wassererneuerung durch die srK sogar größer als durch den kontinuierlichen Wasserstrom. Beide Mechanismen der Wassererneuerung bedingen entsprechend einen Wechsel zwischen der kontinuierlichen und diskontinuierlichen Zufuhr von Nahrungsstoffen.

Ob den srK beim Nahrungserwerb der Ascidien eine allgemeine Bedeutung zukommt, bedarf der Bestätigung, da sie bislang nur für einige wenige Arten nachgewiesen wurden. Zu dem Phänomen selbst können hier keine neuen Beobachtungen angeführt werden, da unsere Untersuchungen in erster Linie auf den Filtrationsvorgang mittels des Kiemenschleimfilters gerichtet waren. So steht auch noch der Nachweis der srK für Clavelina aus, während sie für Ciona von $\mathrm{BACQ}(1934,1935)$ nachgewiesen sind.

Am eigentlichen Mechanismus des Nahrungserwerbs selbst ändert aber die Existenz der srK grundsätzlich nichts, da auch die Nahrungsstoffe, die durch sie in den Kiemendarm gelangen, einzig und allein durch das Kiemenschleimfilter aufgefangen werden können. Nur erhält der Ablauf des Filtrationsvorganges und des Nahrungserwerbs durch die srK den Charakter eines rhythmischen, diskontinuierlichen Vorgangs, da jede Kontraktion notwendig eine Unterbrechung des Nahrungserwerbs bedeutet. Denn nach unseren Beobachtungen an der durchsichtigen Clavelina wird das Kiemenschleimfilter durch eine Kontraktion, wie sie durch äußere Reize hervorgerufen wird, stark in Mitleidenschaft gezogen, was in gleicher Weise auch bei den srK eintreten muß. Teilweise wird es mit dem ausgespritzten Wasser ausgestoßen (vg1. JøRGENSEN und GOLDBERG 1953, pag. 486); in jedem Fall wird es deformiert und muß mindestens z. T. kollabieren und verkleben. Daher kann es seine Funktion nicht mehr voll erfüllen und muß nach Beendigung einer srK in der anschließenden Phase der Erzeugung des kontinuierlichen Wasserstromes neu gebildet werden. Der Filtrationsvorgang kann erst dann wieder vollständig wirksam werden, wenn beide Schleimgewebe über die Seitenflächen des Kiemendarmes geführt und an der Dorsalseite im Sammelvorgang in ganzer Länge vereinigt sind. Solange das nicht der Fall ist, muß notwendig während eines wenn auch nur kurzen Zeitraumes ein Teil der durch die srK und durch den wieder einsetzenden kontinuierlichen Wasserstrom in den Kiemendarm gebrachten Partikel durch die Kiemenspalten hindurch und aus dem Egestionssipho wieder ausgestrudelt werden. Die sich an der Dorsalseite bildende Futterwurst dagegen wird von den srK nicht wesentlich betroffen, da sie von den gekrümmten Dorsalzungen festgehalten wird.

Durch die Existenz der srK ergibt sich für den Ablauf des Nahrungserwerbs die Reihenfolge:

a) kontinuierlicher Wasserstrom - voll ausgebildetes endloses Kiemenschleimfilter - vollständige Filtration;

b) $\mathrm{srK}$ - Unterbrechung des kontinuierlichen Wasserstromes und des Filtrationsvorganges - Wasserausstoßen - Zerstörung des Schleimfilters; 
c) Wassereinsaugen nach srK - neu beginnender kontinuierlicher Wasserstrom - beginnende Neubildung des Kiemenschleimfilters - teilweise Filtration;

d) wie a).

Danach scheinen die srK durch die ständige Unterbrechung des auf Kontinuität abgestellten Filtrationsvorganges den Nahrungserwerb an sich nicht unbedingt rationeller zu gestalten.

Hoyle (1953, pag. 559) schreibt: "It is therefore probable that the principal function of the spontaneous activity in Phallusia is to subserve feeding." Andererseits haben die srK für Phallusia, die als Küstenform in zeitweilig stark getrübtem Wasser lebt, nach Hoyle auch die weitere Bedeutung, eine Anhäufung von unbrauchbarem Material im Kiemendarm zu verhindern und erfüllen damit die gleiche Funktion, die auch die durch äußere Reize, z. B. grobe Partikel, ausgelösten Kontraktionen haben. Indes ist mit beiden Funktionen nicht restlos in Ubereinstimmung zu bringen, daß die Frequenz der srK von der Konzentration der Nahrungspartikel abhängig ist, in der Weise, daß sie bei Nahrungsmangel, d. h. bei g e ringe r Suspensionsdichte stark zunimmt. An sich wäre zu erwarten gewesen, daß gerade bei gesteigerter Suspensionsdichte eine Zunahme der Frequenz eintreten würde, wie es ja auch CarbrsLe (1950) für die Salpen angibt, und was vor allem im Hinblick auf die Reinigungsfunktion der srK sinnvoll wäre. Daher erscheinen weitere Untersuchungen über ihr Vorhandensein und ihre Bedeutung bei möglichst vielen Ascidienarten als notwendig.

2. Es wurde bei der Besprechung der Formbildung des Schleimtrichters bereits kurz darauf hingewiesen (s. o. S. 68 f.), daß seine Ausbildung bei den Jungtieren von Clavelina weniger von den mit Spalten besetzten Flächen des Kiemendarmes bestimmt wird als vielmehr vom Flimmerbogen. Dabei wurde auch schon erwähnt, daß diese Beobachtung einen Vergleich mit dem Nahrungserwerb der anderen Gruppen der Tunicaten, der Copelata und Thaliacea, erleichtert, die ja dem gleichen Ernährungstyp angehören.

Wenn dieser Vergleich im einzelnen noch etwas näher ausgeführt werden soll, so kann er sich nicht auf die zusätzliche Filtrationseinrichtung beziehen, die die Copelata in ihrem Gehäuse besitzen. Das kompliziert gebaute Gehäuse (Lohmann 1899, vgl. Körner 1952) dient bekanntlich der ersten Filtration und ermöglicht dem darin sitzenden Tier die Sonderung aller Partikel nach der Größe, so daß nur die sehr kleinen Partikel, die die Filtereinrichtungen des Gehäuses passieren, in den Kiemendarm gelangen. Der hier stattfindende Vorgang des definitiven Nahrungserwerbs, der allein den entsprechenden Vorgängen bei den Ascidien und Salpen vergleichbar ist, vollzieht sich in einer Weise, die mit der oben für die Jungtiere von Clavelina beschriebenen weitgehend übereinstimmt. Zum Beleg sei mit Abb. 14 die von Fol (1872. Textfig. 5) gegebene schematische Darstellung des Nahrungserwerbs von Oicopleura spissa angeführt.

Die Abbildung läßt zunächst den Bau des Pharynx erkennen, der mit dem Vorhandensein des Endostyls, des Flimmerbogens, des Retropharyngealbandes und eines Paares Kiemenöffnungen alle wesentlichen Organe aufweist, die auch die junge Clavelina besitzt. Nur eine besondere Ausbildung der Dorsalfalte fehlt bei Oicopleura, da sich bei ihr keine Dorsalzungen bzw. keine gesonderte Hautfalte, sondern nur ein aus den beiden Hälften des Flimmerbogens hervorgehendes Flimmerband findet. Die Ubereinstimmung in der Art 
des Nahrungserwerbs kommt wohl am eindeutigsten in der Trichterform des Schleimfilters zum Ausdruck. Fol $(1872,1876)$ läßt allerdings den Schleimtrichter bei Oicopleura weniger aus zwei regelrechten Schleimgeweben entstehen, als vielmehr aus einzelnen fransenartigen Schleimfäden, die erst durch die Tätigkeit des Oesophagus zu dem Trichter zusammengefügt werden, während er bei den Salpen von vornherein aus zusammenhängenden Schleimmembranen gebildet werden soll. Es ist aber sehr wahrscheinlich, daß hier kein

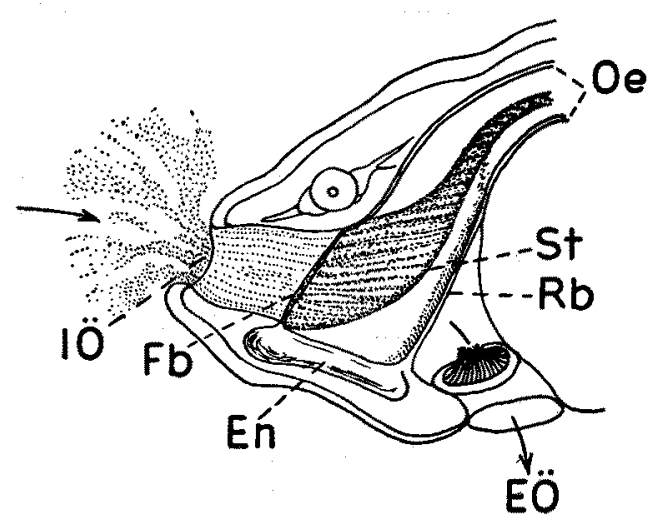

Abb. 14. Oicopleura spissa, Seitenansicht des Vorderkörpers nach Fol (1872, Textfig. 5). En Endostyl, EO Egestionsöffnung = linke Kiemenöfnung, Fb Flimmerbogen, IO Ingestionsöffnung, Oe Oesophagus, Rb Retropharyngealband, St Schleimtrichter.

prinzipieller, sondern nur ein gradueller Unterschied vorliegt. Das für die Formgestaltung des Schleimtrichters bei Oicopleura wichtigste Organ ist offenbar der Flimmerbogen, in dem der Trichter im Pharynx aufgehängt ist, wie es oben auch für die Jungtiere von Clavelina beschrieben wurde.

Auch bei den Thaliacea beruht der Nahrungserwerb auf einem echten Filtrationsvorgang mittels eines trichterförmigen Schleimfilters, wie ebenfalls zuerst FoL (1876) gezeigt hat, und wie später von Fedele (1921, 1923, 1933) und Carlisle (1950) näher untersucht wurde. Die Abb. 15 nach Fedele (1933, fig. 13, vgl. CARLIsLe 1950, fig. 3) läßt die Einzelheiten bei Salpa democratica mit ausreichender Deutlichkeit erkennen, so daß sich eine eingehende Durchführung des Vergleichs erübrigt. Ein grundsätzlicher Unterschied besteht bei den Salpen nur im Mechanismus der Erzeugung des Wasserstromes, der nicht durch Wimperschlag, sondern allein durch Muskeltätigkeit erzeugt wird. Nach Fedele wird der Schleimtrichter bei den Salpen nur während der Perioden "besonders intensiver Ingestion" gebildet, während sonst nur Schleimfäden vom Endostyl erzeugt und über die Flächen des Kiemendarmes transportiert werden sollen (vgl. Ihle 1935). Carlisle (1950) konnte indes zeigen, daß damit ebenfalls kein prinzipieller, sondern nur ein gradueller Unterschied gegeben ist, der sich auf die Intensität der entsprechenden Vorgänge bezieht.

3. Durch seinen spezifischen Mechanismus erhält der Nahrungserwerb der Ascidien den Charakter eines bis zu einem gewissen Grade automatisch ablaufenden Vorganges, der sich immer dann vollzieht, wenn das Tier ungestört ist und den kontinuierlichen Atemwasserstrom erzeugt. Damit dieser komplexe Vorgang in der normalen Weise vor sich gehen kann, bedarf es der koordinierten Zusammenarbeit aller Organe, der Bildungs-, Transport- und Sammelorgane. In einem genau aufeinander abgestimmten Tempo, in dem im Endostyl 
die Schleimgewebe erzeugt werden, müssen sie über die Seitenflächen des Kiemendarmes geführt und an der Dorsalseite zur Futterwurst eingerollt werden, die in einem entsprechenden Tempo in den Oesophagus aufgenommen werden muß. Auf die Rolle, die der vom Wimperschlag der Kiemenspalten erzeugte Wasserstrom dabei spielt, wurde schon kurz hingewiesen: a) führt er die Nahrungsbestandteile heran, b) drückt er die Schleimgewebe auf die Transportvorrichtungen, so daß diese überhaupt erst in Funktion treten können.

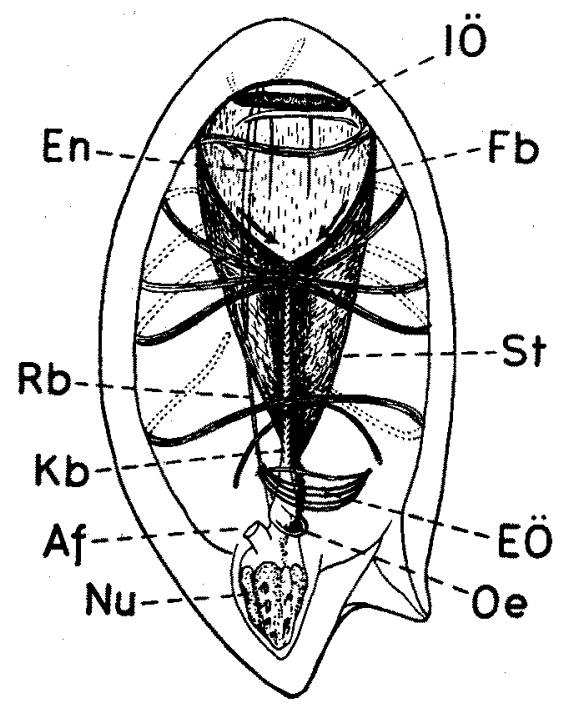

Abb. 15. Salpa democratica, Dorsalansicht nach FEDELE (1933, fig. 13), leicht umgezeichnet. Af After, En Endostyl, EO Egestionsöffnung, Fb Flimmerbogen, IO Ingestionsöffnung, Kb Kiemenbalken, $\mathrm{Nu}$ Eingeweidenukleus, Oe Oesophagus, $\mathrm{Rb}$ Retropharyngealband, St Schleimtrichter.

Über den mit der Erzeugung des Wasserstromes und dem ungestörten Ablauf des Filtrationsvorganges zusammenhängenden nerven- und sinnesphysiologischen Fragenkomplex wissen wir nur wenig. MAcGinitre (1939b, pag. 443) gibt an, daß der Wasserstrom zirkulieren kann, ohne daß gleichzeitig der Filtrationsvorgang stattfindet (,A current of water is maintained through the branchial cavity almost continually, whether the animal is feeding or not"). Das würde bedeuten, daß beide Vorgänge nicht ständig miteinander gekoppelt sein müssen und daß die Drüsen- und Wimperzellen des Endostyls mit den Wimperzellen der Kiemenspalten keine untrennbare funktionelle Einheit bilden. Nach unseren eigenen Beobachtungen gehen Wasserstromerzeugung und Filtrationsvorgang normalerweise parallel, vorausgesetzt, daß die Suspensionen nicht zu dicht und ihre Partikel nicht zu groß sind. Dementsprechend konnte bei den durchsichtigen Clavelinen beobachtet werden, daß sich bei ungestörten Tieren an der Dorsalseite ständig der Nahrungsfaden bildet, der das Ergebnis des Filtrationsvorganges ist. Auf die Reaktion der Ascidien beim Einstrudeln gröberer unerwünschter Substanzen wurde bereits hingewiesen (s. o. S. 60). Die gleiche Reaktion der ruckartigen Kontraktion und des Ausstoßens des gesamten Inhaltes des Kiemendarmes kann auch bei genügend dichten Suspensionen von feinen Partikeln eintreten, wie oft beobachtet wurde und bereits von vaN WeEL (1940) und von Jørgensen und Goldberg (1953, vgl. HoYLE 1953) beschrieben ist. 
Indessen ist denkbar, daß der Filtrationsvorgang bei normalen ungestörten Tieren abhängig vom Füllungszustand des Magens ist. For (1876) beschreibt, daß bei allen von ihm untersuchten Tunicaten die Nahrungsaufnahme beendet wird, wenn der Magen angefüllt ist. $\mathrm{Da}$ aber die Wasserstromerzeugung zur Deckung des $\mathrm{O}_{2}$-Bedarfs weitergehen muß, so ist die Existenz eines entsprechenden Regulationsvermögens nicht von der Hand zu weisen, das vermutlich nur bei länger andauerndem reichlichen Angebot von Nahrungsstoffen wirksam wird. Ein solches Regulationsvermögen könnte für die Ascidien von besonderer Bedeutung sein, da ihnen die Fähigkeit fehlt, unbrauchbare Stoffe regelmäßig in Schleim zu hüllen und als Pseudofaeces aus dem Kiemendarm vor der Aufnahme in den Verdauungstraktus auszuscheiden, eine Eigenschaft, wie sie unter den Wasserstromfiltrierern besonders die Lamellibranchier und unter den Prosobranchiern die Familie Calyptraeidae entwickelt haben. $\mathrm{Ob}$ die Ascidien tatsächlich in der Lage sind, den Filtrationsvorgang, d. h. primär die Schleimfilterbildung willkürlich unter Beibehaltung der Wasserstromerzeugung abzuschalten, muß durch weitere Beobachtungen geklärt werden. Ein experimenteller Hinweis kann in der Reaktion von Clavelina auf Betäubungsmittel gesehen werden. Setzt man dem Seewasser vorsichtig einige Kristalle von Kokain (Cocainum hydrochloricum) in langsam sich steigernder Menge zu, so läßt sich der Effekt erzielen, daß der Filtrationsvorgang abgestoppt wird, obwohl die Wimpern der Kiemenspalten weiterschlagen und den Wasserstrom erzeugen. Das kann mit Hilfe des Suspensionsversuches leicht nachgewiesen werden, da die Partikel jetzt nicht mehr im Kiemendarm aufgefangen, sondern aus dem Egestionssipho wieder ausgestrudelt werden.

Bei den kokainbetäubten Tieren konnte mehrfach eine weitere bemerkenswerte Beobachtung gemacht werden. Bei ihnen kann nämlich der Mageninhalt, der aufgeknäuelte Nahrungsfaden, wieder durch den Oesophagus in den Kiemendarm ausgestoßen werden, was durch eine Rotation des Nahrungsfadens in der der ursprünglichen entgegensetzten Richtung bewirkt wird. Eine ähnliche Beobachtung hat FEDELE (1933) für die Salpen beschrieben, wo sie als Normalerscheinung während der Perioden der "intensiven Ingestion" auftrat.

Weiterhin ist ungeklärt, ob die Intensität des Filtrationsvorganges, d. h. praktisch die Bildungsgeschwindigkeit und Menge des im Endostyl erzeugten Schleims sowie die Schlagfrequenz der am Transport- und Sammelvorgang beteiligten Wimperzellen von der Suspensionsdichte abhängig sind und sich mit wechselnder Suspensionsdichte ändern. Ebenso ist unbekannt, welches das reizaufnehmende Organ ist, über das die Ascidien gegebenenfalls die $\mathrm{Zu}$ fuhr wechselnder Suspensionsmengen mit der entsprechenden Reaktion beantworten. Zum Vergleich sei erwähnt, daß nach CarussLe (1950) die hier gestellte Frage für die Salpen zu bejahen ist, da sich bei ihnen der Filtrationsvorgang mit steigender Suspensionsdichte verstärkt; ferner vermutet er in dem dorsalen Flimmerorgan den Reizrezeptor (vgl. Carlisle 1951). Auch Fol. (1876) hat schon angegeben, daß die Schleimabscheidung des Endostyls gesteigert ist, wenn im eingestrudelten Wasser reichlich Nahrungsbestandteile vorhanden sind. In diesem Zusammenhang ist von besonderem Interesse, daß nach Hoyle (1953) bei Phallusia mammillata die Frequenz der spontanen rhythmischen Kontraktionen vom Nahrungsgehalt des Mediums abhängig ist (s. o. S. 83). Die Frage nach dem Regulationsmechanismus führt Hoyle zu der gleichen Vermutung, daß das dorsale Flimmerorgan der Reizrezeptor ist. 
Einer Prüfung bedarf auch die Frage, ob die Ascidien beim Nahrungserwerb über ein qualitatives Auswahlvermögen verfügen. Daß sie auf mechanische, thermische und chemische Reize sehr empfindlich reagieren, ist allgemein bekannt (vgl. u. a. Hecht 1918). Hoyce (1953) konnte für Phallusia ein gewisses Unterscheidungsvermögen nachweisen, da Hungertiere mit ihrer erhöhten Frequenz der spontanen rhythmischen Kontraktionen nicht auf Suspensionen von kolloidalem Graphit, wohl aber von Futterkulturen mit einer Herabsetzung der Frequenz reagierten. Im übrigen scheint bei den Ascidien für die normalerweise und in ihrer natürlichen Umgebung aufgenommenen Suspensionsbestandteile kein ausgeprägtes Auswahlvermögen zu bestehen, das über ihre Fähigkeit hinausgeht, in der bereits geschilderten Weise auf Suspensionen mit Partikeln verschiedener Größe oder verschiedener Dichte zu reagieren. Insbesondere müssen normalerweise alle Partikel, die überhaupt vom Schleimfilter aufgefangen und in die Futterwurst eingefügt werden, zwangsläufig ohne Rücksicht auf Brauchbarkeit oder Unbrauchbarkeit in den Verdauungstraktus aufgenommen werden. FEDELE (1933) hat für die Salpen ebenfalls festgestellt, daß sie beim Nahrungserwerb kein qualitatives Auswahlvermögen erkennen lassen. In dieser Hinsicht unterscheiden sich die Ascidien von den Wasserstromfiltrierern unter den Prosobranchiern, da für Crepidula gezeigt werden konnte, daß der Inhalt der Futtertasche und die in der Futterrinne gebildete Futterwurst nicht wahllos in den Mund aufgenommen werden, sondern nur dann, wenn sie sich nach Prüfung als brauchbar erweisen (WERner 1953 a). Da den Ascidien, wie erwähnt, auch die Fähigkeit fehlt, unbrauchbare Stoffe als Pseudofaeces bereits vor der Aufnahme in den Verdauungstraktus auszuscheiden, nehmen sie mit den brauchbaren auch stets sehr viele unverdauliche Stoffe auf. Das hat zur Folge, daß ihr Nahrungsbedarf und ihre Koterzeugung entsprechend hoch sind. Wo daher Ascidien in größeren Mengen auftreten, spielen sie eine erhebliche Rolle im Stoff- und Sedimenthaushalt des Meeres.

Die Lösung des Problems des Stoffumsatzes der Ascidien hat bei der Art ihres Nahrungserwerbs die Ermittlung der Filtrationsrate, d. h. der pro Zeiteinheit durch den Kiemendarm hindurchgepumpten Wassermenge zur Voraussetzung. Sie beträgt nach Hecrrt (1918) für eine mittelgroße Phallusia nigra etwa $7,21 / \mathrm{Std}$. Nach Jørgensen ist für Ciona intestinalis bei einer Temperatur von $16-19^{\circ} \mathrm{C}$ eine durchschnittliche Filtrationsrate von $0,231 / \mathrm{Std}$., für Molgula manhattensis von $0,541 / \mathrm{Std}$. je g Nettogewicht anzusetzen ${ }^{1}$ ); dabei hat JøRGENSEN die Filtrationsrate photometrisch aus der Konzentration der Suspensionen bestimmt, die durch die Filtrationstätigkeit verringert wird. Das bedeutet, daß die "filtration rate" praktisch durch die "feeding rate" gemessen wird. Die Anwendung dieser Methode ist berechtigt, wenn Wasserstromerzeugung und Filtrationsvorgang gekoppelt sind (s. o. S. $85 \mathrm{f}$.). Jørgensen und GoLDBERG (1953) konnten zeigen, daß das der Fall ist, wenn die Konzentration der Suspensionen nicht zu hoch gewählt wird.

Die Ermittlung der Filtrationsrate kompliziert sich bei den Ascidien, bei denen zu dem kontinuierlichen Wasserstrom die diskontinuierliche Wassererneuerung durch die spontanen rhythmischen Kontraktionen (srK) hinzu-

1) Diese Werte wurden uns yon Herrn Dr. JøRgensen, Kopenhagen, liebenswürdigerweise brieflich (5. 3. 1954) mitgeteilt, der sie auf Grund früher veröffentlichter Untersuchungen (1949 a) berechnet hat (vgl. auch JøRGENSEN 1952). 
kommt, die wie erwähnt bei Phallusia mammillata nach Hoyle (1953) ein größeres Volumen hat. Hoyle hat mit einer direkten Methode, bei der in der benützten Apparatur eine Kanüle in einen der beiden Siphonen eingeführt wird, den kontinuierlichen Wasserstrom gemessen. Aus seinen Werten von $1 \mathrm{~cm}^{3} / \mathrm{Min}$. bzw. $4 \mathrm{~cm}^{3} / \mathrm{Min}$. (bei $14^{0} \mathrm{C}$ ) läßt sich ein durchschnittlicher Wasserdurchfluß von $0,06 \mathrm{l} / \mathrm{Std}$. und ein Maximum von $0,24 \mathrm{l} / \mathrm{Std}$. errechnen. Die Wassererneuerung im Kiemendarm durch die srK ermittelt HoYLE zu durchschnittlich $0,09 \mathrm{l} / \mathrm{Std}$., so daß er für das Gesamtvolumen des in den Kiemendarm durch beide Mechanismen aufgenommenen Wassers zu dem durchschnittlichen Betrag von $0,15 \mathrm{l} / \mathrm{Std}$. je Tier gelangt. Wenn auch die Kritik berechtigt ist, die HoYle an der Zuverlässigkeit des wahrscheinlich zu hoch ermittelten Wertes von Неснт (s. o.) übt, so erscheinen seine eigenen Werte doch auch im Vergleich zu denen von JøRGENSEN als unverhältnismäßig niedrig, was möglicherweise auf die von Hoyle angewandte Methode zurückzuführen ist. Denn es ist zu bedenken, daß bei der indirekten Methode, wie sie JørGENSEN angewendet hat, in jedem Fall das gesamte durch den Kiemendarm hindurch gepumpte Wasservolumen bestimmt wird, das auf die beiden Mechanismen der Wassererneuerung zurückgeht. Allerdings muß die indirekte Methode bei den Arten, bei denen die srK eine Rolle spielen, notwendig mit einer gewissen Ungenauigkeit behaftet sein, die sich aus der periodischen Unterbrechung des Filtrationsvorganges und der Zerstörung des Schleimfilters ergibt (s. o. S. 82 f.); andererseits kann sich diese Ungenauigkeit nur in dem Sinne auswirken, daß die Filtrationsrate eher zu niedrig als zu hoch bestimmt wird. Daher erscheinen weitere Untersuchungen notwendig, um die Filtrationsrate durch einen Vergleich der mittels der direkten und indirekten Methode möglichst an ein und demselben Individuum gewonnenen Werte zu ermitteln.

Bemerkenswert erscheint das Ergebnis von Jørgensen (1949a), daß die Filtrationsrate bei Wasserstromfiltrierern ganz verschiedener Tierstämme, nämlich von Schwämmen, Muscheln und Ascidien annähernd innerhalb der gleichen Größenordnung liegt, wenn sie bezogen wird auf die vorhandene Menge der Körpersubstanz, als deren Maß der Stickstoffgehalt bestimmt wird (mi/Std. je mg $\mathrm{NH}_{2}-\mathrm{N}$ ).

Die Menge der aufgenommenen Nahrung ist allgemein abhängig a) von der Filtrationsrate, b) von der Suspensionsdichte (Huus 1933). Nach JørgenSEN (1952) ändert sich die Filtrationsrate nicht mit der Suspensionsdichte, solange diese nicht zu hoch ist. Nur bei Hungertieren ist nach Hoyle (1953) der kontinuierliche Wasserstrom verringert, nimmt aber bei Futterzugabe bald wieder seine normale Geschwindigkeit an. Die Menge der aufgenommenen Nahrung und damit der Stoffumsatz hängen daher normalerweise in erster Linie von der Suspensionsdichte ab. Als Grundlage für den Stoffumsatz ermittelt JøRGensen (1952) die Filtrationsrate pro $\mathrm{ml} \mathrm{O}_{2}$-Verbrauch und berechnet daraus den Nahrungsbedarf für den Betrebs- und Aufbaustoffwechsel zu etwa $0,15 \mathrm{mg}$ verwertbarer organischer Substanz pro Liter durchgepumpten und abfiltrierten Wassers.

Die Frage nach der Größe des Stoffumsatzes ist bei den mit Schleimfiltern arbeitenden Wasserstromfiltrierern von besonderer Bedeutung, weil sie die Prüfung der unteren Grenzen des Größenbereichs der aufgefangenen Partikel notwendig macht. Diese Prüfung ist deswegen wichtig, weil ungeklärt ist, ob auch die im Meerwasser enthaltenen submikroskopischen Partikel und die organischen Stoffe in kolloidaler Verteilung aufgenommen werden, 
die an Menge ein Mehrfaches der „geformten" Partikel ausmachen. JørgenSEN (1952), der diese Frage diskutiert, zieht aus seinen Untersuchungen und Berechnungen die Schlußfolgerung, daß die Filtrationsrate groß genug ist, um den Nahrungsbedarf allein mit der Aufnahme der geformten Partikel $(\geqq 0,001 \mathrm{~mm})$ decken zu können. Indes erscheint die Aufnahme von Kolloiden bei den Wasserstromfiltrierern mit ihren sehr feinen engmaschigen Schleimfiltern nicht von vornherein als ausgeschlossen. Durch die Verwendung von Suspensionen bekannter Partikelgröße hat MacGrnitıe (1945) die Maschenweite der Schleimfilter des Echiuriden Urechis caupo und des Polychaeten Chaetopterus variopedatus ermittelt, die näherungsweise mit $40 \AA \mathrm{E}=4 \cdot 10^{-6}$ mm bestimmt wurde. Nach Kalle (1945) haben Kolloide die Größenordnung von $10^{-3}$ bis $10^{-8} \mathrm{~mm}$, Moleküle von $10^{-3}$ bis $10^{-6} \mathrm{~mm}$. Danach sind diese Tiere in der Lage, mit ihren Schleimfiltern noch Kolloide und Moleküle aufzufangen.

Jørgensen (1952) gibt für die Größe der von Ciona abfiltrierten Partikel den unteren Grenzwert von 0,001 $\mathrm{mm}$ an, was dem oberen Grenzwert der Kolloide entsprechen würde. In Ubereinstimmung damit haben JøRGENSEN und Goldberg (1953) gezeigt, daß Suspensionen von kolloidalem Graphit. mit einer Partikelgröße von 0,001 bis $0,002 \mathrm{~mm}$ fast vollständig aufgefangen wurden, während die Eiweißmoleküle der Suspensionen von Hämocyanin und Hämoglobin nur zu einem sehr geringen Prozentsatz zurückgehalten wurden. Durch weitere Untersuchungen muß geprüft werden, ob der von Jørgensen angegebene untere Grenzwert für die Größe der abfiltrierten Partikel bei allen Ascidien der gleiche ist. Beim Filtrationsvorgang von Clavelina konnte von uns beobachtet werden, daß der Nahrungsfaden an seinem hinteren Ende vor der Aufnahme in den Oesophagus oft eine bräunliche Färbung hatte, auch wenn der Wasserstrom frei von mikroskopisch erkennbaren Partikeln war. Danach ist anzunehmen, daß noch submikroskopische Bestandteile aufgefangen wurden, die dem farblosen Schleim des Filters bzw. dem in diesem Falle zum größten Teil aus Schleim bestehenden Nahrungsfaden diese Färbung verliehen.

\section{E. Zusammenfassung}

1. Durch Lebendbeobachtung und Anwendung des Suspensionsversuches wurde der Mechanismus des Nahrungserwerbs der Ascidien einer vergleichenden Analyse unterzogen. Die Untersuchungen wurden in erster Linie an Ciona intestinalis und Clavelina lepadiformis durchgeführt; einige vergleichende Beobachtungen wurden außerdem an Molgula tubifera, Ascidiella aspersa und Phallusia mammillata gemacht.

2. Die Grundvorgänge des Nahrungserwerbs sind:

a) die aktive Erzeugung des Wasserstromes mittels des Wimperschlages der Kiemenspalten und b) seine Filtration.

3. Beim Filtrationsvorgang sind $\mathrm{zu}$ unterscheiden:

a) eine unechte Filtration in der Mundöffnung durch die Reuse der Mundtentakel; hier werden die gröberen Stoffe aufgefangen, die durch die Kontraktionen des Kiemendarmes wieder ausgestoßen werden;

b) eine echte Filtration im Kiemendarm mittels des Kiemenschleimfilters, das nach dem Prinzip des endlosen Schleimfilters arbeitet und die feineren Suspensionsbestandteile abfiltriert. 
4. Der Nahrungserwerb im Kiemendarm läßt sich in folgende Teilvorgänge aufgliedern: a) die Bildung des Schleimfilters, b) sein Transport über die Fläche der Kieme, c) seine Aufrollung im Sammelvorgang, d) die Aufnahme der Futterwurst in den Oesophagus.

5. Das Bildungsorgan für das Schleimfilter ist der Endostyl. Seine Bauelemente sind Drüsen- und Wimperzellen, die zu mehreren Drüsen- und Wimperzellstreifen angeordnet sind. Der Endostyl besteht aus zwei spiegelbildlich gleichen Hälften, die je eine funktionelle Einheit darstellen und je ein Schleimgewebe erzeugen. Die zahlreichen langen, dicht stehenden geißelartigen Cilien des medianen Endostylgrundes bilden eine Längstrennwand für das Lumen des Endostyls. Sie verhindert, daß die von jeder Endostylhälfte gebildeten Schleingewebe in Berührung treten und verkleben.

6. Die beiden Schleimgewebe werden im anschließenden Transportvorgang über die rechte und linke Fläche des Kiemendarmes geführt. Die Transportorgane sind: a) die Wimperstreifen an den oberen Rändern des Endostyls, b) der Flimmerbogen, c) die Flimmerreifen bzw. die Kiemenpapillen im Bereich der mit Spalten versehenen Flächen des Kiemendarmes, d) das Retropharyngealband. Der Transportvorgang stellt die eigentlich wirksame Filtrationsphase dar, während der die beiden Schleimgewebe die vom Atemwasserstrom mitgeführten Partikel vor seinem Durchtritt durch die Kiemenspalten auffangen.

7. In dem anschließenden Sammelvorgang werden die zur Dorsalseite beförderten Schleimgewebe wie zwei Teppiche aufgerollt und zu einer Futterwurst vereinigt. Das Sammelorgan ist die Dorsalfalte bzw. die Gesamtheit aller Dorsalzungen, die zur rechten Seite umgebogen sind und so eine Futterrinne entstehen lassen. In dieser Futterrinne wird primär das rechte Schleimgewebe aufgerollt, in das sekundär das über die konvexe linke Seite der Dorsalfalte bzw. der Dorsalzungen herübergeführte linke Schleimgewebe eingefügt wird. Beide Schleimgewebe bilden daher einen sich ständig erneuernden Schleimtrichter bzw. einen Schleimsack, der in seiner Form von dem Kiemendarm bestimmt wird.

8. Die Futterwurst wird vom Oesophagus aufgenommen und als ganzes in den Magen befördert.

9. Die spontanen rhythmischen Kontraktionen, die für mehrere Ascidienarten nachgewiesen sind, und ihre Bedeutung für die Wassererneuerung und den Nahrungserwerb werden im Anschluß an die Arbeiten von HoYLe (1952, 1953) besprochen.

10. Der Nahrungserwerb der Ascidiacea wird mit dem der Copelata und Thaliacea verglichen. Er vollzieht sich auch bei diesen Gruppen als echter Filtrationsvorgang mittels eines endlosen trichterartigen Schleimfilters.

11. Die mit dem Nahrungserwerb der Ascidien zusammenhängenden physiologischen Fragen werden besprochen.

\section{F. Literaturverzeichnis}

B acq, Z. M., 1934: Ablation du ganglion nerveux chez une ascidie (Ciona intestinalis). C. $r$. Soc. Biol. Paris 117.

- 1935: Observations physiologiques sur le coeur, les muscles et le système nerveux d'une ascidie, Ciona intestinalis. Arch. Int. Physiol. 40.

Berrill, N. J., 1950: The Tunicata. With an account of the british species. London, 
v. Budd en brock, W., 1952, 1953: Vergleichende Physiologie. I. Sinnesphysiologie. II. Nervenphysiologie. Birkhäuser, Basel.

Bü ck mann, A., 1926: Copelata. Grimpe-Wagler, Tierwelt der Nord- und Ostsee, XII a 1.

Carlisle, D. B., 1950: Alcune osservazioni sulla meccanica dell' alimentazione della Salpa. Pubbl. Staz. Zool. Napoli 22.

- 1951: On the hormonal and neural control of the release of gametes in ascidians. J. Exp. Biol. 28.

Fedele, M., 1921: Sulla nutrizione degli animali pelagici. I. Ricerche sui Doliolidae. Mem. R. Com. Talass. Ital. 78.

- 1923: Le attività dinamiche ed i rapporti nervosi nella vita dei Dolioli. Pubbl. Staz. Zool. Napoli 4.

- 1933: Sulla nutrizione degli animali pelagici. III. Ricerche sui Salpidae. Boll. Soc. Nat. Napoli 45

Florey, E., 1951: Reizphysiologische Untersuchungen an der Ascidie Ciona intestinalis L. Biol. Zentralbl. 70.

Fo1, H., 1872: Etudes sur les Appendiculaires du détroit de Messine. Mém. Soc. Phys. Hist. Nat. Genève 21.

- 1876: Über die Schleimdrüse oder den Endostyl der Tunicaten. Morph. Jahrb. 1.

Hagmeier, A., 1951: Die Nahrung der Meerestiere. Hdbch. d. Seefischerei Nordeuropas I. $5 \mathrm{~b}$.

Hecht, S., 1918: The physiology of Ascidia atra Lesueur. I. General physiology. II. Sensory physiology. J. Exp. Zool. 25.

Hoyle, G., 1952: The response mechanism in ascidians. J. Mar. Biol. Ass. Plymouth 31.

- 1953: Spontaneous squirting of an ascidian, Phallusia mammillata Cuvier. Ibid.

Huus, J., 1933: Ascidiacea. Grimpe-Wagler, Tierwelt der Nord- und Ostsee XII a 3.

- 1937: Ascidiacea. Kükenthal-Krumbach, Hdbch. d. Zool. 5.

I h le, J. E. W., 1913: Die Appendicularien. Ergebn. u. Fortschr. d. Zool. 5.

- 1927: Thaliacea. Grimpe-Wagler, Tierwelt der Nord- und Ostsee XII a 2.

- 1937: Desmomyaria. Kükenthal-Krumbach, Hdbch. d. Zool. 5.

- und Ihle-Landenberg, M. E., 1930: Anatomische Untersuchungen über Salpen I. Zool. Anz. 91.

- 1937: Über den Endostyl der Salpen. Festschr.. E. Strand 3.

Jorgensen, C. B., 1949 a: Feeding-rates of Sponges, Lamellibranchs and Ascidians. Nature 163.

- 1949 b: The rate of feeding by Mytilus in different kinds of suspensions. J. Mar. Biol. Ass. Plymouth 28.

- 1952: On the relation between water transport and food requirements in some marine filter feeding Invertebrates. Biol. Bull. 103.

- und Goldberg, E. D., 1953: Particle filtration in some ascidians and lamellibranchs. Ibid. 105.

Kalle, K., 1945: Der Stoffhaushalt des Meeres. Leipzig, Akad. Verlagsgesellschaft.

Körner, W. F., 1952: Untersuchungen über die Gehäusebildung bei Appendicularien (Oicopleura dioica Fol). Z. Morph. u. Okol. d. Tiere 41.

Lacaze-Duthicrs, H., 1874: Les Ascidies simples des côtes de France. Arch. Zool. Expér. 3.

Lohmann, H., 1899: Das Gehäuse der Appendicularien, sein Bau, seine Funktion und seine Entstehung. Schr. Naturw. Verein Schlesw.-Holst. 11.

MacGinitie, G. E., 1937: The use of mucus by marine plankton feeders. Science 86.

- 1939 a: The method of feeding of Chaetopterus. Biol. Bull. 77.

- 1939 b: The method of feeding of the Tunicates. Ibid.

-. 1945: The size of mesh openings in mucous feeding nets of marine animals. Ibid. 88.

- und MacGinitie, N., 1949: Natural history of marine animals. New York, London, Toronto.

Michaelsen, W., 1930: Seescheiden oder Ascidien. Dahl, Tierwelt Deutschlands 17.

Orton, J. H., 1913: The ciliary mechanisms on the gill and the mode of feeding in Amphioxus, Ascidians, and Solenomya togata. J. Mar. Biol. Ass. Plymouth 10.

Plough, H. H. und Jones, N., 1939: Ecteinascidia tortugensis, species nova. With a review of the Perophoridae (Ascidiacea) of the Tortugas. Carnegie Inst. Wash. Publ. 517.

Roule, M. L., 1884: Recherches sur les Ascidies simples des côtes de Provence (Phallusiadées). Ann. Mus. Hist. Nat. Marseille, Zool. 2. 
Seeliger, O., 1885: Die Entwicklungsgeschichte der socialen Ascidien. Jen. Zeitschr. f. Naturw. N. F. 11.

- und Hartmeyer, R., 1893-1911: Tunicata (Manteltiere). Bronn's Kl, u. Ordn. d. Tierreichs 3, Suppl.

Sokólsk a, J, 1931: Contributions à l'histologie de l'endostyle des Ascidies. Fol. Morph. 3. van Wee1, P. V., 1940: Beiträge zur Ernährungsbiologie der Ascidien. Pubbl. Staz. Zool. Napoli 18.

Werner, B., 1951: Ober dic Bedeutung der Wasserstromerzeugung und Wasserstromfiltration für die Nahrungsaufnahme der ortsgebundenen Meeresschnecke Crepidula fornicata L. (Gastropoda Prosobranchia). Zool. Anz. 146.

- 1953 a: Über den Nahrungserwerb der Calyptraeidae (Gastropoda Prosobranchia). Morphologie, Histologie und Funktion der am Nahrungserwerb beteiligten Organe. Helgol. Wiss. Meeresunters. 4.

- $1953 \mathrm{~b}$ : Ausbildungsstufen der Filtrationsmechanismen bei filtrierenden Prosobranchiern Verh. Dtsch. Zool. Ges. 1952.

Yamaguchi, Z., 1931: Some notes on the physiology of Styela clava Herdmann. Sci. Rep. Tôhoku Imp. Univ. 6. 